\title{
A!
}

This is an electronic reprint of the original article.

This reprint may differ from the original in pagination and typographic detail.

Backholm, Matilda; Bäumchen, Oliver

\section{Micropipette force sensors for in vivo force measurements on single cells and multicellular microorganisms}

Published in:

Nature Protocols

DOI:

$10.1038 / \mathrm{s} 41596-018-0110-x$

Published: 01/02/2019

Document Version

Peer reviewed version

Please cite the original version:

Backholm, M., \& Bäumchen, O. (2019). Micropipette force sensors for in vivo force measurements on single cells and multicellular microorganisms. Nature Protocols, 14(2), 594-615. https://doi.org/10.1038/s41596-0180110-x

This material is protected by copyright and other intellectual property rights, and duplication or sale of all or part of any of the repository collections is not permitted, except that material may be duplicated by you for your research use or educational purposes in electronic or print form. You must obtain permission for any other use. Electronic or print copies may not be offered, whether for sale or otherwise to anyone who is not an authorised user. 


\title{
Micropipette force sensors for in vivo force measurements on single cells and multicellular microorganisms
}

\author{
Matilda Backholm ${ }^{1, *} \&$ Oliver Bäumchen ${ }^{2, *}$ \\ ${ }^{1}$ Department of Applied Physics, Aalto University, Puumiehenkuja 2, FI-02150 Espoo, Finland. \\ ${ }^{2}$ Max Planck Institute for Dynamics and Self-Organization (MPIDS), Am Faßberg 17, D-37077 Göttingen, Germany. \\ *Corresponding authors. Correspondence should be addressed to M.B. or O.B. (matilda.backholm@,aalto.fi and \\ oliver.baeumchen@ds.mpg.de).
}

KEYWORDS micropipette, force measurement, biomechanics, motility, micro-swimming, cell adhesion, $C$. elegans, micropipette force sensor, MFS, micropipette deflection

Up to three primary research articles where the protocol has been used and/or developed:

1. "Viscoelastic properties of the nematode Caenorhabditis elegans, a self-similar, shear-thinning worm", M. Backholm, W. S. Ryu, and K. Dalnoki-Veress, Proc. Natl. Acad. Sci. USA 110, 4528-4533 (2013).

2. "The effects of viscosity on the undulatory swimming dynamics of C. elegans", M. Backholm, A. K. S. Kasper, R. D. Schulman, W. S. Ryu, and K. Dalnoki-Veress, Phys. Fluids 27, 091901 (2015).

3. "Adhesion of Chlamydomonas microalgae to surfaces is switchable by light", C. T. Kreis, M. Le Blay, C. Linne, M. M. Makowski, and O. Bäumchen, Nature Physics 14, 45-49 (2018). 


\begin{abstract}
Measuring forces from the $\mathrm{pN}$ to the $\mathrm{mN}$ range is of great importance for the study of living systems from a biophysical perspective. The use of flexible micropipettes as highly sensitive force probes has been established in the biophysical community, advancing our understanding of cellular processes and microbial behavior. The micropipette force sensor (MFS) technique relies on measuring the forces acting on a forcecalibrated, hollow glass micropipette by optically detecting its deflections. The MFS technique covers a wide micro- and mesoscopic regime of detectable forces (tens of $\mathrm{pN}$ to $\mathrm{mN}$ ) and sample sizes ( $\mu \mathrm{m}$ to $\mathrm{mm}$ ), does not require gluing of the sample to the cantilever, and allows for simultaneous optical imaging of the sample throughout the experiment. Here, we provide a detailed protocol of how to manufacture and calibrate the micropipettes as well as how to successfully design, perform and troubleshoot MFS experiments. We exemplify our approach using the model nematode C. elegans, but by following this protocol, a wide variety of living samples, ranging from single cells to multicellular aggregates and millimeter-sized organisms, can be studied in vivo - with a force resolution of down to $10 \mathrm{pN}$. A skilled (under)graduate student can master the technique in about 1-2 months. The whole protocol takes about 1-2 days to finish.
\end{abstract}

\title{
INTRODUCTION
}

Mechanical forces in the $\mathrm{pN}$ to $\mathrm{mN}$ range are ubiquitous in living, biological systems. These forces govern the elasticity $[1,2,3,4]$, motion $[5,6,7]$, and adhesion $[8,9,10]$ of single biomolecules, cells, and multicellular microorganisms. By developing techniques to directly quantify these forces, our understanding of mechanobiological and biophysical processes has been greatly improved on a fundamental level. In the micropipette force sensor (MFS) technique, the spring-like deflection of a long $(0.5-5 \mathrm{~cm})$, thin $(5-100 \mu \mathrm{m})$, and hollow glass micropipette is used to measure or apply forces as low as $10 \mathrm{pN}[11,12,4,9]$. The main principle of the MFS technique is beautifully simple: the spring-like property of an elastic glass micropipette is calibrated by applying a known force $F$ to its end. By optically measuring the pipette deflection $\Delta x$, the Hookean spring constant can be determined as $k=F / \Delta x$. The force-calibrated micropipette can then be used as a sensitive and direct force probe or applicator with high positional ( $\sim \mathrm{nm}$ through the use of a closed-loop piezo drive [9]) and temporal (>1000 fps through the use of a high-speed camera [9]) resolution. During an experiment, the camera captures both the pipette deflection as well as the sample and its surrounding, which permits for simultaneous tracking of sample properties (e.g. shape [7], deformation [4], and configuration of cellular appendages [9]) and potential changes in the environment (e.g. fluid flow [13] and proximity to other objects [14]) while measuring forces. This type of sample imaging during a typical force-displacement cycle is usually not possible in conventional force measurement approaches, such as force-distance curves using atomic force microscopy (AFM). In particular, the fact that the micropipette is deflected in the imaging plane is unique, in comparison to commercially available integrations of AFM and optical microscopy, for which the force measurement is performed perpendicular to the imaging plane. In MFS experiments, living samples can furthermore easily be studied at physiological conditions since all measurements typically 
are performed in a liquid cell, allowing for truly in vivo studies of biological samples using living microorganisms as well as individual cells or cellular aggregates (extracted from higher level organisms) in aqueous media. Since the micropipettes are made in the lab by the researcher, they can be custom designed and manufactured exactly according to the specific experiment at hand. This makes the MFS technique very adaptable and cheap in comparison to for example AFM, where each cantilever usually has to be purchased commercially.

Due to the geometric dimensions of the cantilever (i.e. the flexible part of the micropipette) and elastic property of the glass, MFS spans about 8 orders of magnitude in the force sensitivity (tens of $\mathrm{pN}$ to several $\mathrm{mN}$ ) and about 3 orders of magnitude in the size of the object ( $\mu \mathrm{m}$ to several $\mathrm{mm}$ ). The MFS technique thus fills an intriguing mesoscopic gap that manifests itself between conventional microscopic force measurement techniques such as AFM, optical tweezers and magnetic tweezers that can be used to study single molecules and cells $[15,16,17,18]$, and macroscopic force probes such as dynamometers and load cells that are used to study bulk properties of tissue $[19,20]$, for example. The micro- to mesoscopic regime covered by the MFS technique and its adaptability to the experimental system allows for versatile studies of micro- and millimeter-sized soft and biological samples. Indeed, the suitability of a force measurement technology for a certain experimental system is often decisive in terms of the feasibility of tackling important research questions. For example, the understanding of gecko adhesion in different environmental conditions has required measurements of forces in the ranges of a few $\mathrm{nN}$ (AFM cantilever, single spatula, [21]) to tens of $\mu \mathrm{N}$ (micro-electro-mechanical systems (MEMS) cantilever, single seta, [22]) and tens of $\mathrm{mN}$ (load cell, setae array, [23]).

In this protocol we describe how to fabricate, calibrate, and use micropipette force sensors for force measurements in the sub- $\mathrm{nN}$ to $\mathrm{mN}$ regime. Using the model nematode C. elegans, we give examples of different cantilever shapes and experimental setups that can be applied to study a wide range of soft, micromechanical systems.

\section{Development of the approach}

The predecessor of the MFS technique described here utilizes a solid glass needle to measure forces [24], and is referred to as "micro-needle mechanical manipulation", "force fiber technique", and "cantilever force sensor". This predecessor has been used extensively to directly probe the elasticity of muscle fibers [25], the surface tension of red blood cells [26], the adhesion force between pairs of cells [27, 28] and cells and surfaces [28], the force of microtubule sliding in flagella [5], the mechanical properties of single actin filaments [1], the forces generated by a single cilium [29] and a kinesin molecule [30], the forces involved in the process of actin-based propulsion [6], as well as the dissipating forces on droplets moving on various surfaces $[31,32,33,34,35]$, just to name a few examples. Recently, a further extension utilizing the deflection of an elastic microplate has been used to study cell mechanics [36, 37]. 
The MFS technique described in this protocol utilizes a hollow micropipette and was first introduced by Francis et al. to directly measure the cell detachment force of a single human blood cell from various glass surfaces [11]. The hollow feature of the pipette ingeniously enables attachment of the cells to the force probe simply by using suction. This small improvement in the experimental design eliminates the need of using invasive and complicated attachment strategies of the sample to the cantilever, such as electrostatic interactions, chemical fixation, bioinspired wet adhesives, or glue [18]. Besides biocompatibility issues, synthetic glues exhibit complex rheological response to forces, which need to be considered when studying the mechanical response of a soft material, such as a cell [38].

The use of hollow micropipettes as MFS was likely inspired by the micropipette aspiration technique, which was pioneered by Mitchinson and Swann [39] and further developed by Evans and coworkers [3, $8,40]$, where a cell is partly aspirated into a hollow micropipette using suction, allowing for measurements of the mechanical properties of the cell as well as its adhesion energies to a surface. Regarding this conceptual feature, the MFS technique shares similarities with other methods such as the patch-clamp technique in electrophysiology, developed by Neher and Sakmann [41, 42], as well as micromanipulation techniques employed in in-vitro fertilization setups. When compared to more standardized force measurement techniques, the MFS approach is applicable to a larger force range as well as more versatile experiments. To this day, micropipette force sensors have been used to directly probe for example cell mechanics [43, 44] and force generation [45], the elasticity of chromosomes [2, $46,47,48]$, the interfacial tension of micrometer-sized droplets [49], the strength and breakup of flocks $[50,51]$, the tensile strength of biofilms [51], the adhesion of single bacterial cells [52], the tension of an elastomer film [53], as well as electrically tunable wetting properties [54]. In a further development of the technique, called "cantilevered-capillary force apparatus" (CCFA), the glass micropipettes were exchanged by less fragile but thicker and stiffer glass capillaries that typically are used as columns for gas chromatography [55]. Due to the higher stiffness of the capillaries, these have a force resolution of $\sim 1 \mathrm{nN}$ which is two orders of magnitude higher than MFS. Using CCFA, the interaction forces between vesicles [56] and food emulsion droplets [57] were investigated. Although MFS and CCFA are very similar, they differ in how the cantilevers are manufactured. This protocol can thus only partly be used for the capillary CCFA approach.

\section{Overview of the Procedure}

In this protocol, we describe the technological and scientific developments that started in the group of K. Dalnoki-Veress at McMaster University, Canada, and later continued in our labs. Here, the MFS technique was first reintroduced under the name of "micropipette deflection" (MD) and employed to investigate the adhesion forces and membrane tension of vesicles and living cells [58, 12]. In the present work, we coin the term "micropipette force sensor" to make the name more descriptive for the general 
scientific community and highlight the conceptual similarities to the well-established force measurement techniques based on AFM.

In the MFS approach, the flexible micropipette is manufactured by pulling it out from a thicker, hollow glass capillary tube using a micropipette puller (Step 1). The end of the thin micropipette is cut open and the cantilever is shaped using hot wires (Steps 2-3). A great advantage of MFS over the solid needle approach is to use the hollow feature of the micropipette in its force calibration. This is accomplished by pushing out a small water droplet through the pipette end and using the droplet as the calibration weight (Steps 9 Option A i-vi). By analyzing the volume of the droplet hanging on the outside of the pipette end as the water evaporates, the varying droplet weight can be determined and compared with the pipette deflection, rendering the spring constant of the micropipette with a very high precision (Steps 9 Option A vii-ix). This calibration procedure represents a significant improvement to previous calibration approaches, where, for example, small weights (e.g. differently sized platinum wires) have been placed on a reference needle, which then has been used to calibrate the needle to be used in the experiment [24]. A problem with this approach is that the reference needle is calibrated in the force and deflection range of thousands of $\mathrm{nN}$ and tens of $\mu \mathrm{m}$, whereas the needle to be used in the actual experiment is calibrated and measures forces and deflections in the sub- $\mathrm{nN}$ and $-\mu \mathrm{m}$ regime. Non-linear force-deflection relationships of the reference needle can render different spring constants in the two different regimes, leading to a systematic error in the absolute force measurements. In our water drop-based calibration approach, the micropipette is calibrated closer to the force-deflection regime that it will measure forces in. Furthermore, the drop-approach gives a continuous set of data as the drop volume changes, whereas only discrete data points can be achieved by hanging weights on the pipette.

\section{Example applications of MFS from our research groups}

We have further developed MFS to perform highly sensitive, in vivo force measurements on the two microorganisms Caenorhabditis elegans and Chlamydomonas reinhardtii. In all our studies, the microorganism is caught by a liquid-filled and force-calibrated micropipette by applying suction. Using MFS, we studied the viscoelastic material properties of the nematode C. elegans by performing threepoint bending measurements on anesthetized worms in physiological condition (Figure 1a). We directly measured the longitudinal stiffness for the first time and the time-resolved, dynamic experiments revealed shear-thinning properties of the model organism [4, 59]. Furthermore, we have used MFS to directly measure the drag forces experienced by a swimming nematode in both the lateral and propulsive direction using a three-dimensional micropipette as force probe (Figure 1b and Supplementary Video 1) [7], allowing for the first direct measurements of the drag coefficients of the microswimmer. We also studied the effects of solid boundaries [14] and fluid viscosity [13] on the swimming dynamics of the worm, as well as the forces involved in its crawling motion on different gel surfaces [10]. Finally, we 
used two micropipettes (not force-calibrated) to hold two swimming nematodes close together in an attempt to probe their hydrodynamic interactions and collective motion. Instead, it turned out that the bodies of the tail-tethered, swimming nematodes overlapped, causing them to form fascinating active tangles (Figure 1c), which we described with an analytical model [60]. The great versatility of MFS enabled a very diverse set of questions to be investigated with micropipettes of many different cantilever designs (bottom panel of Figure 1; two and three straight corner bends in two or three dimensions) and spring constants $(k=1-10 \mathrm{nN} / \mu \mathrm{m}$ in the bending and swimming projects, $k=60-350 \mathrm{nN} / \mu \mathrm{m}$ in the crawling study), all of which were designed and made by the (under)graduate students in the lab. All measurements were performed with living nematodes in vivo and combined the data from the force measurements with the simultaneous imaging of the worm bodies. To the best of our knowledge, no other conventional force probe would have suited for this set of work, indicating the vast possibilities of MFS in the biophysical field.

The Bäumchen lab studies the microalga Chlamydomonas reinhardtii, a eukaryotic, unicellular microorganism featuring two flagella that allow for propelling the cell via a breaststroke-like beating in a liquid environment. Besides swimming in a liquid medium, the cells are also able to perform flagellamediated gliding on solid surfaces. By means of MFS experiments, we discovered that the adhesion of Chlamydomonas to surfaces, that is the transition from the planktonic (freely swimming) to the surfaceassociated state, is switchable by light [9]. By controlling the orientation of the cell during MFS experiments, we found that only the flagella may adhere to the substrate, while the cell body does not exhibit any adhesiveness. The fact that the MFS technique is based on the optical imaging of the micropipette deflection, and that it can be combined with contrast-enhancing imaging modes, for example fluorescence, phase contrast, dark field imaging, immersion oil objectives etc., allows for imaging the cell in close proximity to the interface and precisely controlling the light conditions in these experiments. For the first time, we were able to quantitatively study in vivo the flagella-mediated adhesion of Chlamydomonas, a photoactive and motile microorganism, to surfaces. Due to the force resolution of the MFS approach of about $10 \mathrm{pN}$, it was possible to record the forces generated by molecular motors, pulling the cell towards the substrate after the adhesive contact between the flagella and the substrate has been established. Holding a living Chlamydomonas cell with a hollow micropipette via suction has been successfully employed to study the flagellar beating patterns in physiological conditions [61, 62, 63], the hydrodynamic flow fields generated by this beating [64], as well as the photocurrents induced by light stimulation [65]. Beyond the application of micropipettes as a tool to immobilize motile cells, MFS enables to measure in vivo forces exerted by the cellular appendages, simultaneously to tracking their shape and dynamics.

Besides these efforts regarding living and even motile cells, we also investigated the adhesive properties of artificial cells. Recently, we employed the MFS technique to measure the adhesion of biotin- 
functionalized polymersomes, that is microcompartments composed of block-copolymers, featuring diameters in the range of several tens of micrometers, to neutravidin-functionalized glass substrates [66]. Such vesicles are rather flexible and may be partially aspired by the micropipette for immobilization. A main advantage of MFS for probing vesicle adhesion to surfaces is the fact that the contact area can be determined simultaneously to measuring adhesion forces.

\section{Additional applications of the method}

In addition to the examples mentioned above, MFS can be adapted to probe the mechanical properties and adhesion forces of almost any micro- to millimeter-sized soft material, either living or non-living. Also adhesion and (lateral) friction forces of hard materials are possible to probe. By applying standardized substrate manipulation protocols (approach, dwell time, and retraction of the substrate) using high-precision piezo drives and simultaneously recording pipette deflection and position of the substrate, force-distance curves can be performed in analogy to AFM force measurements [9]. Such force-distance curves may be used to probe mechanical cell properties, active cell shape contractions or cellular adhesion to a substrate. Furthermore, designing a sample holder onto which multiple substrates can be mounted, allows for quantitatively comparing adhesion forces of the same living cell at different types of surfaces and to discriminate the relevant intermolecular forces at work (e.g. hydrophilic/hydrophobic, short/long-ranged, electrostatic interactions) [67]. By using a stiff holding pipette instead of a substrate, stretching experiments of macromolecules, aggregates, and droplets can be performed in the absence as well in the presence of external stimuli (e.g. an electric field). Various types of other supports, such as a simple support made of a thick glass micropipette (Figure 1a), can be used for bending measurements of microorganisms and soft materials, in general. To measure swimming, interaction, or drag forces, only the force-calibrated micropipette(s) holding on to the swimmer(s) is needed (Figure 1bc) together with high-speed imaging.

\section{Limitations}

The force limit and sensitivity of the MFS are set by the optical detectability of the pipette deflection and the level of noise caused by mechanical vibrations of the cantilever. By using highly flexible micropipettes $(k \sim 0.1 \mathrm{nN} / \mu \mathrm{m})$, eliminating vibrations by placing the entire setup onto an active antivibration table, and employing a cross-correlation image analysis of the pipette deflection to achieve a sub-pixel deflection resolution, a force sensitivity of about $10 \mathrm{pN}$ can be achieved. In our experiments at this force resolution limit, the imaging of the micropipette is usually performed using a 40x objective (long working distance, NA 0,6) in bright-field configuration without any additional contrast enhancement (using the cameras listed in the materials section). Although this is not the main purpose of the technique, also high forces in the $\mathrm{mN}$ range can be measured by using rather stiff, that is short and thick, micropipettes. The deflection of the micropipette always needs to remain within the linear force-deflection 
regime (typically about $10 \%$ of the cantilever length) in order to avoid non-linear force-deflection features.

The MFS technique is mainly developed to probe micro- to millimeter-sized objects that can be caught with the micropipette. To avoid sucking the entire sample into the pipette, the sample needs to be larger than the minimum inner diameter of a micropipette (typically $\sim 5-10 \mu \mathrm{m}$ ). As described in detail in the procedure of this protocol, the micropipette manufacturing process enables to adapt the micropipette geometry to the requirements of the sample of interest and even fabricate thinner $(1-5 \mu \mathrm{m})$ micropipette openings for the study of micrometer-sized objects, such as single bacteria. The MFS experiments are commonly performed with samples in suspensions, but a sample can also easily be studied in its surfaceassociated state $[9,11]$.

The throughput of the method can be estimated from the number of different cells/organisms that can be measured in a certain time frame. An experienced user may set up the experiment and study about 5-10 different cells/organisms of one kind (each typically contributing 10 measurement cycles) in one working day. This limitation, however, strongly depends on the experience level of the user and also on how easy it is to capture a cell or organism in the desired configuration. In that sense the MFS technique is comparable to bacterial force measurements, however, the number of force cycles per cell is usually significantly higher in AFM force spectroscopy due to automated protocols and fast ramp rates of commercial instruments.

Lastly, the main conceptual limitation of the MFS technique lies in the fragility of the glass micropipettes. These are thinner than a strand of hair and may break at careless handling. A skilled experimentalist who is detail oriented and careful will learn how to work with the micropipettes without breaking them. If this expertise level is not achieved, an excessive amount of time will be spent on continuously making and calibrating new pipettes instead of performing actual experiments with the technique. A learning period of 3-6 months is to be expected to reach such an expert level. Mastering the MFS technique to its full extent opens exciting research avenues and allows for pushing the force sensitivity limits, where the highly sensitive glass micropipettes provide a force resolution that is two orders of magnitude better than the capillaries used in CCFA [55] and a signal-to-noise ratio that is comparable to force measurements using AFM.

\section{Experimental design}

Microscope setup. This approach requires an inverted optical microscope is with 5-100X objectives (typically featuring a long working distance) and a high-resolution and possibly high-speed scientific camera to capture the experiment. A large bit depth (ideally 12-16 bit) as well as a small pixel size of the camera chip appears highly beneficial for high-quality imaging and a high-resolution detection of the 
micropipette's deflection. The camera is controlled by a computer using, for example, software provided by the camera manufacturer or, alternatively, self-made LabView scripts to collect the images. LabView may also be used to drive the motorized linear translational stages, in case they are needed for the experiment (e.g. for force-distance curves). For high-resolution, high-speed image recordings we suggest a posteriori image processing and data transfer to the computer via a broadband connection, e.g. Camera Link. In order to damp environmental vibrations, the whole setup should preferably be mounted on an active antivibration table, which may reside on a passive optical table or breadboard. The remaining sources of micropipette fluctuations originate from building vibrations and/or acoustic noise, for example from camera fans, vacuum pumps, lab doors being opened or closed and other mechanical devices in the lab. In addition, a cage or hood might be built around the entire setup to isolate the experiment from external stimuli. Note that the latest generation of inverted microscopes (e.g. the IX-83 by Olympus, Japan) come with the option of controlling the microscope by external haptic elements, which may be beneficial for noise-sensitive MFS experiments since, once the experiment is set up and ready to go, there is no need to further manually touch the experiment for operating the microscope. All experiments are viewed from below the liquid cell (see examples of different setup possibilities in the bottom panel of Figure 1). Depending on the experiment at hand, a $x$ - or $x y$-translational motor stage is mounted on the left hand side, which should exhibit both coarse $(\sim 5 \mathrm{~cm}$ range) and fine-tuning ( $\mu \mathrm{m})$ abilities to easily maneuver the substrate (or any other feature) to the desired position. For recording force-distance curves (in analogy to AFM force measurements) adding another piezo-driven stage is desirable to achieve a higher position resolution of the substrate. The right hand side consists of a micropipette holder for mounting the micropipette. Here, we use a three-axis piezo-driven micromanipulator, which is also commonly used, for example in in vitro fertilization applications. For the experiment, plastic tubing and a plastic syringe are needed to first fill the micropipette with liquid and then apply suction to hold on to the sample. When connecting the liquid-filled micropipette to a liquid reservoir, the user may optionally connect the system to a microfluidic pump, which enables more precise pressure control inside the micropipette as compared to a manually operated syringe.

Micropipette design. In general, the micropipette design should be adapted to the experimental requirements. If very small forces in the range of several tens of $\mathrm{pN}$ to a few $\mathrm{nN}$ are to be probed (e.g. typical propulsion forces of microorganisms as well as cell adhesion forces), the spring constant of the micropipette should be small, that is $k<1 \mathrm{nN} / \mu \mathrm{m}$. Such small spring constants can be achieved by making a very long $(3-5 \mathrm{~cm})$ and thin $(10-20 \mu \mathrm{m})$ cantilever. On the other hand, if the experiment will probe larger forces (e.g. $100 \mathrm{nN}$ in the bending of a stiff rod), the spring constant should be tuned accordingly $(\sim 10-20 \mathrm{nN} / \mu \mathrm{m})$. To achieve optimal force sensitivity, high pipette flexibility is beneficial. However, the stiffer the pipette, the smaller the noise in the force data. In other words, there is a tradeoff between high force sensitivity and low noise when choosing the spring constant. The micropipette should also be shaped according to the requirements of the experiment (see examples in Figure 1). If the force 
of interest is oriented perpendicular to the end part of the pipette (pulling the end to the side as illustrated in Figure 2a), a completely straight pipette can be used. Most often, however, the force to be measured is oriented parallel to the end of the pipette (pulling it forward or pushing it backwards as shown in Figure 2b). In that case, a straight corner bend is needed so that the long middle part of the pipette acts as the cantilever and deflects under the applied force, whereas the first short part only acts to hold the sample in the right orientation. It is important that the short part is significantly shorter than the long part so that deflection occurs only in the cantilever. As shown in Figures 1a and $\mathbf{c}$, additional straight corner bends can be made to simplify the mounting of the pipette into the chamber and to bring the ends of two parallel micropipettes very close to each other. As illustrated in Figure 1b, it is also possible to, for example, measure two perpendicular forces at the same time by making straight corner bends in three dimensions. The long cantilever will then deflect in two orthogonal directions and the perpendicular force components can be measured by detecting the deflection of the two short bends at the end of the pipette. In this case it is necessary to calibrate the spring constants in both orthogonal directions.

Micropipette manufacturing. In this protocol we describe how to pull micropipettes out of a thicker (1 $\mathrm{mm}$ ) hollow glass capillary using a micropipette puller (Step 1). The end of the pulled micropipette is cut using a microforge and the cantilever is finally shaped according to the requirements of the experiment using the hot filament in the microforge (Steps 2-3). Investing in the equipment necessary and learning how to manufacture the micropipettes provides a great level of independence for performing a wide variety of biomechanical experiments with very different micropipettes. A cheaper (short term) alternative to using an own micropipette puller is to buy pre-pulled micropipettes that are commercially available. The user would, however, still need to shape (and calibrate) the pulled micropipettes according to the requirements of the experiment.

Micropipette calibration. The micropipette has to be calibrated in the same direction as the acting force in the experiment. A straight pipette can be assumed to have the same stiffness in all deflection directions, whereas there might be small differences in the spring constant of a bent pipette due to the small torques contributed by the short pipette parts. In this protocol, we describe a water drop-based calibration technique (Step 9 Option A), where a small water drop is pushed out from the liquid filled pipette so that the water drop is supported by the hydrophilic glass pipette. The added weight of the drop causes a deflection of the micropipette due to the gravitational force. By gradually changing the droplet volume (by pushing in more liquid or letting the drop evaporate), and thus changing the added weight, and recording the pipette deflection as a function of drop volume, the spring constant of the pipette can be determined ("calibration.m" code provided as Supplementary Software 1). The calibrated pipette can then either be used directly in an experiment or to calibrate another pipette (Step 9 Option B). When comparing the two calibration approaches, calibration Option B can be used to calibrate a batch of micropipettes in a row. However, a higher level of uncertainty is connected with this approach, as any 
miscalibration of the reference pipette may propagate into an entire batch of micropipettes. In order to save time, calibration Option B can be performed on the force-sensing micropipette right before the actual experiment is to be performed. In this way, the newly calibrated micropipette does not have to be dismounted before the experiment. An alternative approach to calibration Option B is to use a commercial force probe, e.g. an AFM cantilever, as pre-calibrated force sensors to calibrate micropipettes that are rather stiff.

Chamber design. The simplest chamber can be built of two parallel glass coverslips separated by spacers and held together with clamps. The spacing between the coverslips needs to be sufficiently small to allow for a stable capillary bridge to be formed between the two walls. Depending on the micropipette design (see above), a different chamber might be needed, as exemplified in Figure 1b, where a threedimensional micropipette was used in a cylindrical chamber. This chamber was built by epoxying a piece of rubber tubing onto a glass coverslip. More advanced chambers can be built by designing a permanent custom-made liquid cell on the basis of a custom-made microscope stage plate [9]. The two glass slides (top and bottom plates) are held in place by custom-made frames (either made from transparent polymethylmethacrylate or opaque stainless steel/aluminum) and pieces of o-rings as spacers. In our force-distance measurements, we adjust the height of the liquid cell, that is the distance between the parallel plates, to $3.4 \mathrm{~mm}$ by choosing appropriate o-ring spacers and tightening the screws to stabilize the liquid cell. A substrate holder, where either one or even multiple substrates can be mounted onto, enters on one side into the liquid cell, whereas a second opening allows for the micropipette to enter on the opposite side. We recommend fabricating a custom-made, hammer-shaped substrate holder from stainless steel that is sufficiently thin $(2 \mathrm{~mm})$ to enter the liquid chamber [9]. For force-distance experiments, we find it useful to work with rather large liquid volumes by adjusting the lateral distance of the o-ring pieces to about 3-4 cm. For such larger liquid volumes, parasitic flows induced by evaporation are negligible and stable measurements can be performed over several hours. This chamber design readily allows for injecting chemical agents into the aqueous solution during an experiment to test the effect of drugs or inhibitors on the cellular properties of the exact same cell. In addition, repeated removal and addition of liquid can be used to successively replace the medium. 
Data analysis. Matlab is used to read in the images from the experiment and analyze the pipette deflection using cross-correlation analysis of the micropipette signature ("deflection.m" code provided as Supplementary Software 2). In this analysis, the one-dimensional intensity profile along a perpendicular line drawn across the micropipette is extracted in each time frame of the movie (Figure 3a). Each profile (Figure 3b) is then compared to the reference profile of the first frame by spatially shifting the two until they match best, as defined by the maximum of the cross-correlation coefficient (Figure 3c). The required spatial shift that maximizes the cross-correlation function corresponds to the deflection $\Delta x$ of the micropipette. This deflection can be determined with sub-pixel resolution, provided the position of the maximum is taken from a fit of an analytical smooth function, for example, a Gaussian curve, to the data or a spline interpolation of the correlation values. The actual force is calculated by multiplying the micropipette deflection $\Delta x$ with the spring constant $k$ (obtained from the micropipette calibration), that is $F=k \Delta x$. The deflection analysis of the experimental data should be performed in approximately the same pipette position as in the calibration experiment (e.g. as close to the pipette end as possible). For quantitative force measurements, it is important to also record the force-free micropipette in order to obtain a reference $(F=0)$ value for the actual measurements. Matlab can also be used to analyze other features of the experimental data, for example the sample position, morphology and/or deformation can be simultaneously tracked by employing digital image processing involving edge detection codes.

\section{Expertise needed to implement the protocol}

A skilled and detail oriented undergraduate student should be able to learn how to use the MFS technique within 1-2 months of work by carefully following this protocol. The micropipettes take several hours to make and calibrate, but are easy to break. A significant part of the training process will thereby involve learning how carefully handle the micropipettes. Based on our hands-on experience, this is the most time consuming aspect in the MFS protocol and can cause hurdles in the experimental work. It takes about 3-6 months of full working time use to reach an expert level where almost no micropipettes break due to carelessness. We stress that once mastered, the technique provides unique research possibilities in a vast range of systems.

\section{MATERIALS}

BIOLOGICAL MATERIALS

- The protocol described here has been used on various biological samples, including tissue culture cells and living organisms (see Introduction for details). We exemplify our procedure using wild-type nematodes (isolate N2 from Bristol, UK) acquired from the Caenorhabditis Genetics Center. 


\section{REAGENTS}

- Deionized water (alternatively: ultra-pure water, Milli-Q ${ }^{\circledR}$ A10 Water Purification System, total organic carbon residues: 5 ppb, resistivity at $25^{\circ} \mathrm{C}: 18.2 \mathrm{M} \Omega \mathrm{cm}$, Merck, Darmstadt, Germany)

- $\quad$ Ethanol (CAS 64-17-5; Carl Roth GmbH, Karlsruhe, Germany; ROTISOLV®, HPLC Gradient Grade, Purity > 99.9 \%)

For the C. elegans study exemplified in this protocol:

- Escherichia coli (Caenorhabditis Genetics Center, OP50) and nematode growth media (NGM) plates (Teknova, Hollister, CA, USA, Cat. No. N1098)

- $\quad$ M9 buffer (made in-house)

\section{EQUIPMENT}

- Inverted microscope (e.g. IX-73 or IX-83, Olympus, Tokyo, Japan)

- Active vibration isolation stage (I4 Series, Accurion, Göttingen, Germany)

- Objectives (5-100X) featuring long working distances $(60 \mathrm{x}-100 \mathrm{x}$ is only used for high-resolution oil immersion imaging to visualize, e.g., cellular appendages such as flagella, otherwise typically $10 \mathrm{x}-40 \mathrm{x}$ is used for MFS.)

- Camera (camera specs should be adapted to the experimental requirements, for example a Grasshopper3 GS3-U3-41C6M, Point Grey Research, Canada and PCO Edge 4.2, PCO, Kelheim, Germany for higher frame rates.)

- Micropipette puller (PN-31, Narishige, Tokyo, Japan or P-97, Sutter Instrument, Novato, CA, USA)

- $\quad$ Microforge (MF-900, Narishige, Tokyo, Japan)

- $\quad$ Piezo-driven xyz-micromanipulator (Burleigh TS-5000-300, Thorlabs, USA) for micropipette positioning

- Linear motor stages (LTA-HS, Newport Corporation, Irvine, CA, USA) for coarse alignment of the substrate

- Multi-axis tilt platforms (M-36, Newport Corporation, Irvine, CA, USA)

- Borosilicate glass capillaries (outer/inner diameter 1/0.75 mm, TW100-6, World Precision Instruments, Sarasota, FL, USA)

- Two micropipette holders (H-7, Narishige, Tokyo, Japan)

- $\quad$ Plastic (PTFE) tubing, inner diameter $1 \mathrm{~mm}$ (CT-1, Narishige, Tokyo, Japan)

- $\quad$ Plastic syringes (with sizes between 2 and $50 \mathrm{~mL}$ )

- Needle to connect the syringe to the plastic tubing

- Glass coverslips (e.g. 2947-75x50, Corning, USA, 75 x 50 mm, 1mm thickness)

- $\quad$ Spacers (home made, the design depends on the experiment at hand)

- $\quad$ Piece of silicon wafer ( 2 × $2 \mathrm{~cm}$, Sigma-Aldrich, CAS Number 7440-21-3) (to be used as a mirror in the calibration)

- $\quad 45^{\circ}$ angled mounting plate (optomechanical component from Thorlabs)

- External light source (for the calibration setup)

- $\quad$ MATLAB software (MathWorks, www.mathworks.com)

- LabView software (National Instruments, www.labview.com)

\section{Optional items:}

- Injection holder set (IM-H1, Narishige, Tokyo, Japan)

- Microgrinder (EG-401, Narishige, Tokyo, Japan)

- High-precision pressure controller designed to handle fluid in microfluidic systems (MFCS-8C, Fluigent, Paris, France)

- Home-built box (cardboard parts and metal railings can be purchased from Thorlabs, USA)

\section{REAGENT SETUP}


- Aqueous-based buffer solution. The composition of the solution depends on the type of biological sample. In the example described in this protocol (C. elegans) we used the buffer M9, which was made in-house using this recipe: $\mathrm{Mix}_{3} \mathrm{~g} \mathrm{KH}_{2} \mathrm{PO}_{4}, 6 \mathrm{~g} \mathrm{Na} 2 \mathrm{HPO}_{4}, 5 \mathrm{~g} \mathrm{NaCl}$, and $1 \mathrm{ml} 1 \mathrm{M} \mathrm{MgSO}_{4}$. Make up to $1 \mathrm{~L}$ with distilled $\mathrm{H}_{2} \mathrm{O}$. Autoclave. The buffer can be stored at $20^{\circ} \mathrm{C}$ for up to 4-6 months.

\section{EQUIPMENT SETUP}

MFS setup. In Figure 4, a photograph of a MFS setup is shown with the different parts denoted with numbers.

\section{PROCEDURE}

Micropipette fabrication TIMING $\sim \mathbf{1 0 - 6 0}$ min (depends on the researcher's level of experience and on the type of pipette, e.g. straight vs. three-dimensional, to be made)

1. Using a micropipette puller, stretch the micropipette from a thick glass capillary (inner/outer diameter $1 / 0.75 \mathrm{~mm}$ ). The pulling settings are chosen based on the desired properties of the pipette. To make a 3-4 cm long and 20-30 $\mu \mathrm{m}$ thick cantilever using the Narishige PN-31 micropipette puller with a preinstalled, rounded filament, the parameters to be used are: magnet main 52, magnet sub 25, heater 90 , and the setscrew at $8 \mathrm{~mm}$. As a rule of thumb, a higher filament temperature and faster pulling speed will render longer and thinner pipettes.

! CAUTION The end of the pulled micropipette is very sharp and care should be taken not to insert it into the body, where micrometer-sized glass fragments may be incorporated and potentially cause health problems.

\section{? TROUBLESHOOTING}

2. Cut the end of the pulled micropipette by placing it against a hot (filament heating setting $\sim 20-30$ on the Narishige microforge) metal wire and looping it around this using a small stick. When the current across the wire is turned off, the quick temperature drop will cause the glass to contract and break. The cut should be made at the desired cantilever length. The end of the micropipette can be heated to remove sharp edges that could cut for example the cell membrane. If a very thin (1-5 $\mu \mathrm{m})$ micropipette opening is desired, move the micropipette (looped around the hot wire) slightly $(\sim 100-300 \mu \mathrm{m})$ away from the wire before turning off the current. This will elongate the softened micropipette at the position of the wire and when the current is turned off, the end will be cut at the new, thinner part of the micropipette.

CRITICAL STEP The opening of the pipette should be smooth (to avoid damaging the sample with a sharp edge) and straight (to align the sample perpendicularly to the cantilever).

\section{? TROUBLESHOOTING}

3. Make a right-angle bend at the desired position by gently placing the straight pipette onto a hot wire (not as hot as during cutting to prevent melting the glass; filament heating setting $\sim 15-20$ on the Narishige 
microforge) and pushing down the part to be bent using a small metal wire. Repeat if multiple bends are needed. To make the three-dimensional micropipette shown in Figure 1b, make the first 90-degree bend very close $(100-200 \mu \mathrm{m})$ to the pipette end. Then rotate the entire pipette by 90 degrees in the pipette holder, so that the next 90-degree bend (positioned $\sim 100-300 \mu \mathrm{m}$ from the first one) can be made perpendicularly to (and out of plane from) the first straight part. The resulting micropipette will have two short, perpendicular parts at the end for imaging the cantilever deflections in these directions, and a long cantilever that is oriented 90 degrees out of plane of the two short bends.

CRITICAL STEP Be careful to make all angles 90 degrees so that the orthogonal force is measured accurately. Strive towards using as low a filament temperature as possible to only soften the glass enough for bending the pipette. Too high temperatures (filament heating setting $>25$ on the Narishige microforge) will melt the glass and make the inner diameter of the pipette smaller, possibly too thin for water to pass through.

PAUSE POINT The manufactured pipettes can be stored for months in a dust-free environment, for example, a class 100 laminar flow workbench. A plastic box with holes drilled in the bottom can be used as a storage container. The micropipettes are placed to stand in the holes and by using unique color-coded numbers written next to each hole, the pipettes can easily be identified. We recommend marking the outside of the thick part of each micropipette with the same number (written in roman numerals for simplicity) and making an electronic list including details about the number, general properties and specific location of each micropipette. Micropipettes that are in use for experiments and filled with water or aqueous solution can be stored in between experiments in fresh, ultra-pure water in a large petri dish (in order to avoid the formation of a meniscus inside the micropipette due to evaporation), which should be sealed with a lid, properly labeled and stored in a dust-free environment, for example, a class 100 laminar flow workbench.

\section{Chamber setup for calibration and MFS experiment TIMIING $\sim 10$ min}

4. Build a chamber using, for example, two parallel cover slips with spacers in between. Mount the chamber onto a thicker microscope glass slide with tape and connect the thicker glass slide to the inverted microscope using magnetic tape glued on both the microscope and glass slide.

CRITICAL STEP For a reusable chamber that is integrated into a microscope stage plate, a more advanced liquid cell setup can be manufactured (see Experimental Design for details).

\section{Micropipette preparation for calibration and MFS experiment TIIMING $\sim 10 \mathrm{~min}$}

5. Attach the micropipette to a micropipette holder.

CRITICAL STEP If the knob is tightened too strongly, the thick, back end of the pipette might break. If this happens, blow off any residual glass dust and reattach the pipette more carefully. If there is any glass dust left at the pipette end when the pipette is filled with liquid, the dust might be pushed into the pipette and 
towards the thin part of the micropipette, where it can form a permanent clog.

6. Carefully put down the micropipette holder on the end of a table so that the micropipette remains feely suspended above the support.

CRITICAL STEP Make sure to monitor the position of the micropipette during the entire experimental procedure to avoid breaking it.

7. Connect tubing to a needle attached to a syringe containing liquid (if possible, use deionized water to avoid clogging of the pipette) and push liquid all the way through the tubing so that there are no air bubbles left. In calibration experiments, use deionized water. For living samples, the use of buffer solution might be required instead.

CRITICAL STEP Since air is compressible, an air bubble caught in the pipette or tubing will deform when suction is applied and greatly reduce the applied negative pressure on the sample, making this difficult to hold on to.

8. Pick up the micropipette holder, attach the liquid-filled tube to the back end of the micropipette, and push liquid through the pipette. Make sure there are no air bubbles trapped in the micropipette.

\section{? TROUBLESHOOTING}

\section{Micropipette calibration}

9. Calibrate the pipettes either using the water-drop approach (Option A) or using an already calibrated micropipette (Option B)

\section{A. Calibration using the water-drop approach TIMING $\sim \mathbf{~ h ~}$}

CRITICAL This approach is illustrated in Figure 5. The calibration is done in air and does therefore not require a chamber.

i. Turn the pipette so that the cantilever is in the horizontal direction (perpendicular to the gravitational force) and mount it above the microscope.

ii. Using, for example, a $45^{\circ}$ angled mounting plate (optomechanical component), mount a mirror (e.g. a clean piece of silicon wafer) at a 45 -degree angle very close to the pipette and use an external light to shine light onto the mirror. See Figure 5a-b where an example using a straight pipette is shown, also bent pipettes can be calibrated with this approach.

iii. View the mirror image of the pipette with the microscope (see example picture in Figure 5c). In this way, vertical pipette deflections can be observed with the inverted microscope. Alternatively, build another setup with a camera mounted so that it images the micropipette from the side. See example pictures in Figure 5d using a bent pipette, also straight pipettes can be calibrated with this approach. 
iv. Focus the objective, start the camera and turn on the anti-vibration table.

v. Push out a water droplet using the syringe so that drop rests on the outside of the pipette (Figure 5d).

vi. Steadily increase the drop volume by pushing in more water and record the change in deflection, $\Delta x$ (Figure 5d). Alternatively, let the drop evaporate while recoding the change in deflection. The evaporation approach typically renderes less noisy data but takes longer time to perform.

vii. Analyze the pipette deflection in Matlab using the "deflection.m"-code (see

\section{Supplementary Software 2).}

viii. Use the "calibration.m"-code (see Supplementary Software 1) to calculate the drop volume $(V)$ by modeling it as an ellipsoid and assuming that the two minor axes are equally long (see the resulting ellipsoidal fits as red lines in Figure 5d). When running the code, include only the part of the experiment where the drop is large enough for the ellipsoidal fit to correctly describe its contour. After subtracting the volume of the glass micropipette inside of the drop, the code plots the calculated drop weight as a function of the micropipette's deflection (Figure 5e) and does a linear fit to the data. The data in this example is linear over three orders of magnitude in force and deflection data. The slope of the fit represents the spring constant of the micropipette and the error corresponds to the $95 \%$ confidence interval of the fit. In the inset of Figure 5e, a zoomin of the data close to origin is shown, highlighting the linearity of the data also at very small drop sizes. In the beginning of the experiment, the drop might not wet the pipette symmetrically (see example in the first picture of Figure 5d). In this particular example, the ellipsoidal fit still captures the outline of the drop accurately and the resulting forcedeflection data follows the linear trend (see inset of Figure 5e). The arrows in the graphs denote the middle picture of Figure 5d (the first time frame of when the drop wets the pipette symmetrically), whereas the two other pictures are from the very beginning and end of the experiment.

\section{? TROUBLESHOOTING}

ix. Repeat Steps v-viii 3-5 times to obtain an average value as well as a standard deviation of the spring constant $k_{\mathrm{s}}$. The relative standard deviation should be less than $10 \%$ and is usually larger than the $95 \%$ confidence interval of the linear fit to the force-deflection data.

x. Unmount and empty the micropipette.

CRITICAL STEP The calibration should be performed carefully to ensure correct force measurements. Any shift in the center of mass of the drop during the calibration experiment should, for example, be minimized. When calibrating a straight cantilever (see example in Figure 5c), this shift is typically smaller than $\sim 200 \mu \mathrm{m}$, which 
corresponds to a $<1 \%$ relative change on a $2.5 \mathrm{~cm}$ long cantilever. For practical force measurement purposes, such a shift is negligible. The error associated with this shift becomes more prominent for stiffer (shorter) micropipettes that need to be calibrated with heavier (larger) water drops. On a micropipette with a short bend at the end (such as the one in Figure 5d), the center of mass remains constant in the direction of the cantilever. When calibrating this type of micropipette, care should be taken that the drop is not positioned at the bending point of the pipette, as this might significantly deform the drop from the assumed ellipsoidal shape.

B. Calibration using an already calibrated micropipette TIIMING $\sim \mathbf{1} \mathbf{h}$ (for one pipette, add $\sim 15$ min per additional pipette to be calibrated)

CRITICAL This approach is illustrated in Figure 6. The calibration is done in liquid and does therefore require a water-filled chamber. Several pipettes can be calibrated using the same setup.

i. Calibrate a (typically straight) pipette as described in Step 9 Option A, determining its spring constant $k_{\mathrm{s}}$.

ii. Completely fill a chamber with deionized water.

iii. Prepare the calibrated and the newly made pipette (with a short bend at the end) as described in Steps 5-8.

iv. Mount the calibrated, straight pipette ("s") on the motorized stage on the left side of the microscope and insert it into the chamber.

v. Mount the bent pipette ("b") on the translational stage on the right of the microscope and carefully insert it into the chamber.

vi. Move the pipettes so that they are in the same focus plane and so that their ends almost touch in the middle of the field of view of the microscope (Figure 6a). Make sure that both pipettes are parallel with the glass slides and do not touch any part of the chamber. Also make sure that the thick parts of the micropipettes and not the thin, cantilever part of the pipette go through the meniscus (i.e. the vertical air-liquid interface).

vii. Make sure there is no flow of water in or out of the pipettes.

CRITICAL STEP If there is liquid flowing out of the bent micropipette, there will be a "liquid cushion" trapped between the two micropipettes at the point of contact, making this contact "soft": the straight micropipette starts deflecting as it pushes towards both the liquid cushion and the bent pipette. If, on the other hand, there is liquid flowing into the bent micropipette, this will suck onto the straight micropipette, visible as a rapid snap-in a bit before actual contact should have occurred. Both of these effects destroy the calibration measurement. One needs to zero the applied pressure in the syringes manually until no soft contact or rapid snap in can be observed.

viii. Turn on the anti-vibration table and start the camera. 
ix. Push the straight pipette into the bent pipettes at a constant speed using the motor.

x. Analyze the deflection of both micropipettes in Matlab using the "deflection.m-code (see Supplementary Software 2).

xi. Plot the straight micropipette's deflection as a function of time (Figure $6 \mathbf{b}$ ) and record the relative deflection $\left(\Delta x_{\mathrm{s}}\right)$ after the point of contact. Then plot the force applied by the straight pipette $\left(F_{\mathrm{s}}=k_{\mathrm{s}} \Delta x_{\mathrm{s}}\right)$ as a function of the deflection of the bent pipette (Figure 6c) and do a linear fit to the data. The slope of this fit is the spring constant of the bent pipette and the error corresponds to the $95 \%$ confidence interval of the fit.

xii. Repeat Steps ix-xi 3-5 times to obtain an average value as well as the standard deviation of the micropipette's spring constant. The relative error should be less than $10 \%$.

xiii. Unmount and empty the newly calibrated pipette. Repeat Steps iii and v-xii with all pipettes to be calibrated.

PAUSE POINT The calibrated pipettes can be stored for months in a dust-free environment (see Step 3). Given the significant amount of time required to manufacture and calibrate a single micropipette, we recommend reusing it as many times as possible instead of continuously making new ones. In theory, there is no maximum limit of how many times a micropipette can be used in an experiment. In practice, what most often happens is that, unless the micropipette breaks, it will become clogged from some impurity sucked in during an experiment or even from tiny glass pieces broken off at the thick, back end of the pipette. An experienced and careful user should be able to use the same micropipette throughout the entire research project (i.e. for more than 6-12 months).

\section{Preparations before the MFS experiment TIIMING $\sim 15$ min}

10. Completely fill the chamber with the desired liquid (e.g., deionized water, buffer solution, culture medium) and the sample to be studied. In the example described here we use M9 buffer solution.

CRITICAL STEP To prevent evaporation of the liquid during the experiment, which, for example, could lead to problematic osmotic effects on the sample, one may add mineral oil on top of the medium or use an environmental chamber to control the temperature and keep the humidity high. By using a large liquid volume in the chamber, one furthermore minimizes the effect of evaporation.

11. If a stiff holding pipette is to be used, manufacture this and prepare it in the same manner as described in Steps 1-2 and 5-8, and mount it on the motorized translational stages on the left of the setup and carefully insert it into the chamber. Alternatively, if a substrate is needed, attach this to a home-built substrate holder and insert it into the chamber. To perform three-point bending measurements similar to that described in Figure 1a, a simple support is needed. To make such a support, pull a micropipette as described in Step 1 and then cut at it a position where the diameter is about $50 \mu \mathrm{m}$ as described in Step 2. Shape the end of the micropipette into a U-shape by bending it half way around a thick $(0.5 \mathrm{~mm}$ in diameter $)$ hot wire. Finally, rotate the micropipette by 90 degrees 
in the pipette holder and bend it by 90 degrees out of the U-plane. The slender structure to be bent in the experiment will be simply supported by the two vertical U-parts of the glass support as shown in Figure 1a. No glue or other attachment strategies are required, unless a very large deformation is desired, which would cause the material to slip against the glass pipette of our suggested support.

12. Mount the micropipette holder with the calibrated micropipette onto the micromanipulator next to the microscope and carefully move the pipette into the chamber, such that it is parallel to the plane of the microscope stage.

13. Move all mechanical parts to the field of view of the microscope and to the middle ( $z$-direction) of the chamber.

CRITICAL STEP Care should be taken that no mechanical parts touch the chamber walls and that a thick part of the force-calibrated pipette passes through the meniscus (i.e. the vertical air-liquid interface) out of the chamber. If a thin part of the micropipette comes into contact with the liquid meniscus, there might be drift in the experiments (as the liquid might evaporate over the course of the experiment at the liquid-air interface) and risk of breaking the pipette. The pipettes should be aligned parallel with the walls and perpendicular to the liquid meniscus in order to minimize the curvature of the liquid-air interface where the micropipette penetrates the liquid.

\section{Performing the MFS experiment TIIMING $\sim 10-60$ min}

14. Carefully move the microscope stage (i.e. the chamber) to find the sample. Do not move the pipette, since it needs to remain in the field of view of the microscope. If the density of the sample is different from the density of the immersing fluid, the pipette needs to be moved in the $z$-direction to be in the same plane as the sample.

15. Use the fine-tuning on the $x y z$-translational stage to carefully approach the sample. When it is very close to the force-calibrated micropipette opening, grab on to the sample by applying suction with the syringe.

CRITICAL STEP The applied pressure depends strongly on the type of sample to be studied, for example immobilizing a swimming C. elegans nematode requires a higher suction than catching a swimming Chlamydomonas microalga. For studying systems that might break or be sucked into the micropipette, e.g. unilamellar vesicles (liposomes or polymersomes), we recommend regulating the aspiration pressure inside the micropipette by attaching a microfluidic pump to the tubing, instead of using a manual syringe. Alternatively, one may also employ the hydrostatic pressure difference between a reservoir and the sample to precisely adjust the aspiration pressure inside the micropipette. For systems demanding more sophisticated pressure control solutions, we redirect the reader to established protocols on micropipette aspiration applications $[67,68,69]$. 


\section{? TROUBLESHOOTING}

16. Move the micropipette and the microscope stage so that the pipette and the sample are in the middle (in $x, y$, and $z$ ) of the chamber to avoid any effects of the walls and the liquid-air meniscus on the measurements.

17. Turn on the anti-vibration table and make sure there is no excessive environmental noise or airflow in the room. Enclosing the entire setup by a home-built hood not only helps to isolate the experiment from acoustic vibrations and air flows (e.g. from air conditioning systems), but also enables controlling the light conditions if working with photosensitive microorganisms. An additional (smaller) incubation chamber is necessary if the temperature or humidity is to be controlled.

18. Perform the experiment (e.g. push a substrate against the sample using the motorized translational stages, or simply observe a swimming microorganism) while continuously capturing image sequences or recording a movie with the camera using a high enough frame rate (e.g. 2 fps in C. elegans bending measurements using a bending speed of $1 \mu \mathrm{m} / \mathrm{s}$, and $56 \mathrm{fps}$ for C. elegans swimming experiments) to render suitable time resolution. Repeat the experiment 5-10 times and with different samples for reproducibility checks and sufficient statistics for biological samples.

19. After the experiment, unmount and empty the micropipette.

PAUSE POINT The micropipettes can be stored in a safe and dust-free location (see Pause Point after Step

3). If a biological experiment has been performed and a buffer solution or a cell growth medium has been used, rinse the micropipette with a solvent (e.g. ethanol).

Image and data analysis TIMING $\sim \mathbf{1 - 2 0}$ min (depends on the number of frames and level of analysis to be done)

20. Analyze the micropipette deflection of the movie using the "deflection.m" Matlab-code (see

\section{Supplementary Software 2).}

\section{? TROUBLESHOOTING}

21. Use customized edge-detection codes (such as "regionprops" in Matlab) to detect the sample shape, to measure various distances of interest, and to track, for example, the deformation of the sample during the force measurement.

\section{TIMIING}

Steps 1-3, Micropipette fabrication: $\sim 10-60 \mathrm{~min}$.

Steps 4, Chamber setup for calibration and MFS experiment: $\sim 10 \mathrm{~min}$. 
Steps 5-8, Micropipette preparation for calibration and MFS experiment: $\sim 10 \mathrm{~min}$.

Step 9 Option A, Micropipette calibration using the water-drop approach: $\sim 1 \mathrm{~h}$.

Step 9 Option B, Micropipette calibration using an already calibrated micropipette: $\sim 1 \mathrm{~h}$.

Steps 10-13, Preparations before the MFS experiment: $\sim 15 \mathrm{~min}$.

Steps 14-19, Performing the MFS experiment: $\sim 10-60 \mathrm{~min}$.

Steps 20-21, Image and data analysis: 1-20 min.

\section{? TROUBLESHOOTING}

Troubleshooting advice can be found in Table 1.

TABLE 1 | Troubleshooting table

\begin{tabular}{|c|c|c|c|}
\hline Step & Problem & Possible reason & Solution \\
\hline 1 & Too long pipettes. & $\begin{array}{l}\text { Wrong micropipette } \\
\text { puller settings. }\end{array}$ & $\begin{array}{l}\text { Use a lower filament temperature } \\
\text { and/or lower pulling speed and/or } \\
\text { shorter setscrew setting. }\end{array}$ \\
\hline 1 & Too short pipettes. & $\begin{array}{l}\text { Wrong micropipette } \\
\text { puller settings. }\end{array}$ & $\begin{array}{l}\text { Use a higher filament temperature } \\
\text { and/or faster pulling speed and/or } \\
\text { longer setscrew setting. }\end{array}$ \\
\hline 2 & Tilted pipette end. & $\begin{array}{l}\text { Incorrect cutting } \\
\text { procedure. }\end{array}$ & $\begin{array}{l}\text { Try not to push the micropipette too } \\
\text { much against the hot wire before } \\
\text { looping the micropipette around it. }\end{array}$ \\
\hline \multirow[t]{3}{*}{8} & $\begin{array}{l}\text { Unable to push liquid } \\
\text { through the pipette. }\end{array}$ & $\begin{array}{l}\text { Not enough pressure } \\
\text { applied. }\end{array}$ & $\begin{array}{l}\text { To push water through the thin end of } \\
\text { the micropipette a high pressure is } \\
\text { required. Use a smaller (e.g. } 2 \mathrm{~mL} \text { ) } \\
\text { syringe to achieve the required } \\
\text { pressure. }\end{array}$ \\
\hline & & Pipette end not open. & $\begin{array}{l}\text { Remember to cut the micropipette end } \\
\text { after pulling the micropipette so that } \\
\text { the end is not closed (see Step 2). }\end{array}$ \\
\hline & & Clogged pipette. & $\begin{array}{l}\text { If possible, use deionized water inside } \\
\text { the pipettes to avoid salts or } \\
\text { impurities to form clogs in the } \\
\text { micropipettes. Rinse the micropipette } \\
\text { with water and empty the micropipette } \\
\text { after an experiment. Be careful to not }\end{array}$ \\
\hline
\end{tabular}


suck in impurities from the chamber during the measurements. An attempt to clean the micropipette can be made using ultrasonication in a deionized water bath.

9Aviii. $\operatorname{Bad} F=k \Delta x$ fit to the data (see examples in Figure 8).
Incorrect zero level (data does not extrapolate through origin).

Noisy data (see Figure 8a).

Non-linear data (see

Figure 8b).

Unable to catch/hold on to sample.
The definition of the force-free micropipette position $(F=0)$ is incorrect due to large micropipette vibrations, drift, or jumps. Please see solutions to these specific problems in Step 20 below. Redo the calibration experiment.

Large micropipette vibrations during the experiment, see suggestions of how to better isolate the setup from noise in Step 20 below.

Try to realign the micropipette and make sure there is no strain on the plastic tubing connected to the pipette. Check if the edge detection of the droplet shape works properly and eventually improve the image contrast and/or the droplet location on the pipette. Make sure the deflection does not exceed $10 \%$ of the cantilever length.

Unmount and empty the pipette and try to suck out the impurity clogging the pipette. Make sure to use clean liquid (preferably deionized water) inside the micropipette to avoid clogs to be formed. If the clog is not removed by emptying the micropipette, see the "Unable to push liquid through the pipette - Clogged 
pipette" troubleshooting above of how to clean the micropipette. Also be careful not to suck in any impurities from the chamber during the measurements and always empty the micropipette after an experiment or store it in a clean liquid bath.

Air bubble(s) inside the tubing or pipette.

Bad isolation.

liquid; $\Delta x> \pm 2 \mu \mathrm{m}$ in air) pipette vibrations (see example in Figure

\section{7a)}

Drift of the pipette

position (does not remain constant although no apparent force is acting on the pipette, see example in Figure $7 \mathrm{~b}$ and Figure 9).
Unmount and empty the micropipette and disconnect the tubing. Push out all air bubbles from the tubing, refill it completely and reconnect it to the pipette and refill this. Make sure no air is trapped anywhere within the pipette or tubing.

Turn on the active anti-vibration table. The entire setup can also be shielded from airflow by building a box around the entire setup. The experiments should always be performed in lab space where noise and vibrations from the environment can be minimized. Basement lab space is preferred over top-level floors, since building vibrations contribute to the low frequency noise level.

Cantilever deflected at If any thin part of the pipette goes the liquid-air meniscus. through the meniscus, there will be capillary forces strong enough to cause deflections, seen as drift in the data. Make sure that a thick part of the pipette exits the chamber through the meniscus. This problem becomes more pronounced in long-term measurements where the meniscus starts to move due to evaporation of 
the liquid: make sure that the entire thin part of the pipette remains far from the meniscus throughout the experiment. Also, try to minimize meniscus curvatures where the micropipette penetrates the liquid-air interface as well as evaporation of the liquid medium.

Tubing pulling on pipette.

Bad mechanical attachment.

Bad vibration isolation.

Slipping between sample and supporting pipette/substrate.
Make sure there is no pulling force acting on the back end of the micropipette by the plastic tubing. If there is any strain in the tubing, this might slowly shift the entire pipette and cause drift in the deflection data. Make sure all mechanical attachments (screws etc.) are properly tightened. Avoid using any soft materials (such as glue or rubber bands) in the mounting of the micropipette, as these will deform when strained.

See "Large vibrations" troubleshooting above.

Perform the experiment slower, with smaller deformations, and make sure all components are aligned to avoid non-perpendicular forces to cause slipping. If too high forces are acting on the sample, it will slip against the support.

\section{ANTICIPATED RESULTS}

In Figure 7, the common problems of (a) bad vibrational isolation and (b) micropipette drift are shown in plots of the micropipette deflection as a function of time. In Figure 7a, the badly isolated setup was placed on a normal, lightweight office desk with no anti-vibration table and with no box shielding the system from airflow and acoustic vibrations. The resulting micropipette deflection is very large (red 
data). By building the setup on a passive optical table with an anti-vibration stage and a cardboard box around the setup, fluctuations of less than $\Delta x \sim \pm 2 \mu \mathrm{m}$ were observed for a freely suspended micropipette in air (blue data). In Figure 7b, the deflection of the same flexible micropipette as in (a) on a properly isolated setup is shown where the problem of drift occurs (red data). Both of these experimental problems are addressed in the troubleshooting table (Table 1, Step 20).

In Figure 8, the results (water drop weight as a function of micropipette deflection) from unsuccessful calibration (as described in Step 9 Option A) attempts are plotted. The experiments were performed with the same micropipette as in the successful experiment highlighted in Figure 5e. In Figure 8a, micropipette vibrations during the experiment resulted in noisy force-deflection data (not as noisy as in Figure 7a). The best linear fit to the data has a relative error that is one order of magnitude higher than in the successful experiment ( $0.2 \%$ vs. $0.03 \%)$. See the troubleshooting table (Table 1, Steps 9Aviii and 20) for suggestions of how to reduce noise. In Figure 8b, the recorded force-deflection data is non-linear. The insets of both graphs show zoom-ins on the data close to origin. Suggestions on how to solve this problem can be found in the troubleshooting table (Table 1, Steps 9Aviii and 20).

In Figure 9, an example data set (force as a function of time) from a (a) successful and (b) unsuccessful C. elegans swimming experiment is plotted. In (b), drift of the pipette causes a continuous decrease in the mean value of the force data, making quantitative force measurements difficult. It can be difficult to tell the drift apart from a "real" physical event taking place in the experiment and we encourage the experimentalist to take care with building the setup and performing the experiment (as discussed in the troubleshooting table: Table 1, Step 20) so that any issues contributing to external drift can be avoided. Depending on the experiment at hand, it might be possible to correct for the drift afterwards in the data analysis. However, if the average of the force data is non-constant as a result of the physical system studied (e.g. in a force relaxation experiment), any additional drift of the pipette cannot be removed during analysis.

\section{AUTHOR CONTRIBUTIONS}

M.B. and O.B. developed the protocol and wrote the manuscript.

\section{ACKNOWLEDGEMENTS}

M.B. gratefully acknowledges the support by the Academy of Finland (Centres of Excellence Programme (2014-2019, grant agreement No 272361) and Postdoctoral Researcher Project (grant agreement No 309237)). O.B. acknowledges funding from the German Research Foundation (DFG) under grant BA3406/2. The authors sincerely thank K. Dalnoki-Veress for inspiring discussions and support. R.D. Schulman, C.T. Kreis, M.M. Makowski, T. Böddeker, and Q. Magdelaine are acknowledged for sharing their hands-on experiences and for valuable technical suggestions regarding improvements of the protocol. 


\section{COMPETEING FINANCIAL INTERESTS}

The authors declare no competing interests.

\section{DATA AVAILABILITY STATEMENT}

The data presented in this protocol is available from the corresponding authors upon request.

\section{REFERENCES}

[1] Kishino, A. \& Yanagida, T. Force measurements by micromanipulation of a single actin filament by glass needle. Nature 334, 74-76 (1988).

[2] Houchmandzadeh, B., Marko, J.F., Chatenay, D. \& Libchaber, D. Elasticity and structure of eukaryote chromosomes studied by micromanipulation and micropipette aspiration. J. Cell Biol. 139, 112 (1997).

[3] Hochmuth, R.M. Micropipette aspiration of living cells. J. Biomech. 33, 15-22 (2000).

[4] Backholm, M., Ryu, W.S. \& Dalnoki-Veress, K. Viscoelastic properties of the nematode Caenorhabditis elegans, a self-similar, shear-thinning worm. Proc. Natl. Acad. Sci. USA 110, 4528-4533 (2013).

[5] Kamimura, S. \& Takahashi, K. Direct measurement of the force of microtubule sliding in flagella. Nature 293, 566-568 (1981).

[6] Marcy, Y., Prost, J., Carlier, M.-F. \& Sykes, C. Forces generated during actin-based propulsion: a direct measurement by micromanipulation. Proc. Natl. Acad. Sci. USA 101, 5992-5997 (2004).

[7] Schulman, R.D., Backholm, M., Ryu, W.S. \& Dalnoki-Veress, K. Dynamic force patterns of an undulatory microswimmer. Phys. Rev. E: Rapid Communications 89, 050701 (2014).

[8] Evans, E.A. Minimum energy analysis of membrane deformation applied to pipet aspiration and surface adhesion of red blood cells. Biophys. J. 30, 265-284 (1980).

[9] Kreis, C.T., Le Blay, M., Linne, C., Makowski, M.M. \& Bäumchen, O. Adhesion of Chlamydomonas microalgae to surfaces is switchable by light. Nat. Phys. 14, 45-49 (2018).

[10] Rabets, Y., Backholm, M., Dalnoki-Veress, K. \& Ryu, W. S. Direct measurements of drag forces in C. elegans crawling locomotion. Biophys. J. 107, 1980-1987 (2014).

[11] Francis, G.W., Fisher, L.R., Gamble, R.A. \& Gingell, D. Direct measurement of cell detachment force on single cells using a new electromechanical method. J. Cell. Sci. 87, 519-523 (1987). 
[12] Colbert, M.-J., Brochard-Wyart, F., Fradin, C. \& Dalnoki-Veress, K. Squeezing and detachment of living cells. Biophys. J. 99, 3555 (2010).

[13] Backholm, M., Kasper, A.K.S., Schulman, R.D., Ryu, W.S. \& Dalnoki-Veress, K. The effects of viscosity on the undulatory swimming dynamics of C. elegans. Phys. Fluids 27, 091901 (2015).

[14] Schulman, R.D., Backholm, M., Ryu, W.S. \& Dalnoki-Veress, K. Undulatory microswimming near solid boundaries. Phys. Fluids 26, 101902 (2014).

[15] Florin, E.-L., Moy, V., \& Gaub, H.E. Adhesion forces between individual ligand-receptor pairs. Science 264, 415-417 (1994).

[16] Neuman, K.C. \& Nagy, A. Single-molecule force spectroscopy: optical tweezers, magnetic tweezers and atomic force microscopy. Nat. Methods 5, 5491-505 (2008).

[17] Dufrene, Y.F., Martinez-Martin, D., Medalsy, I., Alsteens, D., \& Müller, D.J. Multiparametric imaging of biological systems by force-distance curve-based AFM. Nature Methods 10, 847-854 (2013).

[18] Beaussart, A. et al. Quantifying the forces guiding microbial cell adhesion using single-cell force spectroscopy, Nat. Protoc. 9, 1049-1055 (2014).

[19] Iivarinen, J.T., Korhonen, R.K., Julkunen, P. \& Jurvelin, J.S. Experimental and computational analysis of soft tissue stiffness in forearm using a manual indentation device. Med. Eng. Phys. 33, 1245-53 (2011).

[20] Parker, D., et al., A device for characterizing the mechanical properties of the plantar soft tissue of the foot. Med. Eng. Phys. 37, 1098-1104 (2015).

[21] Huber, G., et al. Evidence for capillarity contributions to gecko adhesion from single spatula nanomechanical measurements. Proc. Natl. Acad. Sci. USA 102, 16293-16296 (2005).

[22] Autumn, K., et al. Adhesive force of a single gecko foot-hair. Nature 405, 681-685 (2000).

[23] Loskill, P., et al. Macroscale adhesion of gecko setae reflects nanoscale differences in subsurface contribution. J.R. Soc. Interface 10, 20120587 (2012).

[24] Shimamoto, Y.\& Kapoor, T.M. Microneedle-based analysis of the micromechanics of the metaphase spindle assembled in Xenopus laevis egg extracts. Nat. Protoc. 7, 959-969 (2012).

[25] Sichel, F.J.M. The elasticity of isolated resting skeletal muscle fibers. J. Cell. \& Comp. Physiol. 5, $21-$ $42(1934)$. 
[26] Norris, C.H. The tension at the surface, and other physical properties of the nucleated erythrocyte. $J$. Cell. \& Comp. Physiol. 14, 117-133 (1939).

[27] Coman, D.R. Decreased mutual adhesiveness, a property of cells from squamous cell carcinomas. Cancer Res. 4, 625-629 (1944).

[28] Coman, D. R. Adhesiveness and stickiness: two independent properties of cell surfaces. Cancer Res. 21, 1436-1438 (1961).

[29] Yoneda, M. Force exerted by a single cilium of Mytilus edulis. I. J. Exp. Biol. 37, 461-468 (1960).

[30] Meyhofer, E. \& Howard, J. The force generated by a single kinesin molecule against an elastic load. Proc. Natl. Acad. Sci. USA 92, 574-578 (1995).

[31] Suda, H., Yamada, S. Force measurements for the movement of a water drop on a surface with a surface tension gradient. Langmuir 19, 529-531 (2002).

[32] Lagubeau, G., Le Merrer, M., Clanet, C. \& Quéré, D. Leidenfrost on a ratchet. Nat. Phys. 7, 395-398 (2011).

[33] Pilat, D.W. et al. Dynamic measurement of the force required to move a liquid drop on a solid surface. Langmuir 28, 16812-16820 (2012).

[34] Daniel, D., Timonen, J.V.I., Li, R., Velling, S.J. \& Aizenberg, J. Oleoplaning droplets on lubricated surfaces. Nat. Phys. 13, 1020-1026 (2017).

[35] Gao, N., et al. How drops start sliding over solid surfaces. Nat. Phys. 14, 191-196 (2018).

[36] Mitrossilis, D., et al. Single-cell response to stiffness exhibits muscle-like behavior. Proc. Natl. Acad. Sci. USA 106, 18243-18248 (2009).

[37] Mitrossilis, D., et al. Real-time single-cell response to stiffness. Proc. Natl. Acad. Sci. USA 107, 1651816523 (2010).

[38] Bowen, W.R., Lovitt, R.W. \& Wright, C.J. Atomic force microscopy study of the adhesion of Saccharomyces cerevisiae. J. Colloid Inter. Sci. 237, 54-61 (2001).

[39] Mitchison, J.M. \& Swann, M.M. The Mechanical Properties of the Cell Surface. J. Exp. Biol. 31, 443460 (1954). 
[40] Evans, E.A. Analysis of adhesion of large vesicles to surfaces. Biophys. J. 31, 425-431 (1980).

[41] Neher, E. \& Sakmann, S. Single-channel currents recorded from membrane of denervated frog muscle fibres. Nature 260, 799-902 (1976).

[42] Sakmann, B. \& Neher, E. Patch clamp techniques for studying ionic channels in excitable membranes. Annu. Rev. Physiol. 46, 455-472 (1984).

[43] Basu, R. et al. Cytotoxic T cells use mechanical force to potentiate target cell killing. Cell 165, 100-110 (2016).

[44] Guillou, L., Babataheri, A., Puech, P.-H., Barakat, A.I., \& Husson, J. Dynamic monitoring of cell mechanical properties using profile microindentation. Sci. Rep. 6, 21529 (2016).

[45] Sawicka, A. et al. Micropipette force probe to quantify single-cell force generation: application to T-cell activation. Mol. Biol. Cell 28, 3229-3239 (2017).

[46] Houchmandzadeh, B. \& Dimitrov, S. Elasticity measurements show the existence of thin rigid cores inside mitotic chromosomes. J. Cell Biol. 145, 215-223 (1999).

[47] Poirier, M., Eroglu, S., Chatenay, D. \& Marko, J.F. Reversible and irreversible unfolding of mitotic newt chromosomes by applied force. Mol. Biol. Cell 11, 269-276 (2000).

[48] Poirier, M.G., Eroglu, S. \& Marko, J.F. The bending rigidity of mitotic chromosomes. Mol. Biol. Cell 13, 2170-2179 (2002).

[49] Moran, K., Yeung, A. \& Masliyah, J. Measuring interfacial tensions of micrometer-sized droplets: a novel micromechanical technique. Langmuir 15, 8497-8504 (1999).

[50] Yeung, A.K.C. \& Pelton, R. Micromechanics: a new approach to studying the strength and breakup of flocs. J. Colloid Interface Sci. 184, 579-585 (1996).

[51] Poppele, E.H. \& Hozalski, R.M. Micro-cantilever method for measuring the tensile strength of biofilms and microbial flocs. J. Microbiol. Methods 55, 607-615 (2003).

[52] Tsang, P.H., Li, G., Brun, Y.V., Freund, L.B., \& Tang, J.X. Adhesion of single bacterial cells in the micronewton range. Proc. Natl. Acad. Sci. USA 103, 5764-5768 (2006).

[53] Schulman, R.D. \& Dalnoki-Veress, K. Liquid droplets on a highly deformable membrane. Phys. Rev. Lett. 115, 206101 (2015). 
[54] 't Mannetje, D., et al. Electrically tunable wetting defects characterized by a simple capillary force sensor. Langmuir 29, 9944-9949 (2013).

[55] Frostad, J.M., Collins, M.C. \& Leal, L.G. Cantilevered-capillary force apparatus for measuring multiphase fluid interactions. Langmuir 29, 4715-4725 (2013).

[56] Frostad, J.M., Seth, M., Bernasek, S.M. \& Leal, L.G. Direct measurement of interaction forces between charged multilamellar vesicles. Soft Matter 10, 7769-7780 (2014).

[57] Frostad, J.M., Collins, M.C. \& Leal, L.G. Direct measurement of the interaction of model food emulsion droplets adhering by arrested coalescence. Colloids Surf. A: Physicochem. Eng. Aspects 441, 459-465 (2014).

[58] Colbert, M.-J., Raegen, A.N., Fradin, C. \& Dalnoki-Veress, K. Adhesion and membrane tension of single vesicles and living cells using a micropipette-based technique. Eur. Phys. J. E 30, 117 (2009).

[59] Backholm, M., Ryu, W.S. \& Dalnoki-Veress, K. The nematode C. elegans as a complex viscoelastic fluid. Eur. Phys. J. E 38, 36 (2015).

[60] Backholm, M., Schulman, R.D., Ryu, W.S. \& Dalnoki-Veress, K. Tangling of tethered swimmers: Interactions between two nematodes. Phys. Rev. Lett. 113, 138101 (2014).

[61] Rüffer, U. \& Nultsch, W. Comparison of the beating of cis- and trans-flagella of Chlamydomonas cells held on micropipettes. Cell Motil. Cytoskeleton 7, 87-93 (1987).

[62] Rüffer, U. \& Nultsch, W. Flagellar photoresponses of Chlamydomonas cells held on micropipettes: II. Change in flagellar beat pattern. Cell Motil. Cytoskeleton 18, 269-278 (1991).

[63] Wan, K.Y. \& Goldstein, R.E. Coordinated beating of algal flagella is mediated by basal coupling. Proc. Natl. Acad. Sci. USA 113, E2784-E2793 (2016).

[64] Drescher, K., Goldstein, R.E., Michel, N., Polin, M., \& Tuval, I. Direct measurement of the flow field around swimming microorganisms. Phys. Rev. Lett. 105, 168101 (2010).

[65] Harz, H. \& Hegemann, P. Rhodopsin-regulated calcium currents in Chlamydomonas. Nature 351, 489491 (1991).

[66] Petit, J. et al. A modular approach for multifunctional polymersomes with controlled adhesive properties. Soft Matter 14, 894-900 (2018). 
[67] Kee, Y.S. \& Robinson, D.N. Micropipette aspiration for studying cellular mechanosensory responses and mechanics. Methods Mol. Biol. 983, 367-82 (2013).

[68] Biro, M. \& Maître, J.L. Dual pipette aspiration: a unique tool for studying intercellular adhesion. Methods Cell Biol. 125, 255-67 (2015).

[69] Guevorkian, K. \& Maître, J.L. Micropipette aspiration: a unique tool for exploring cell and tissue mechanics in vivo. Methods Cell Biol. 139, 187-201 (2017).

\section{FIGURES}

a
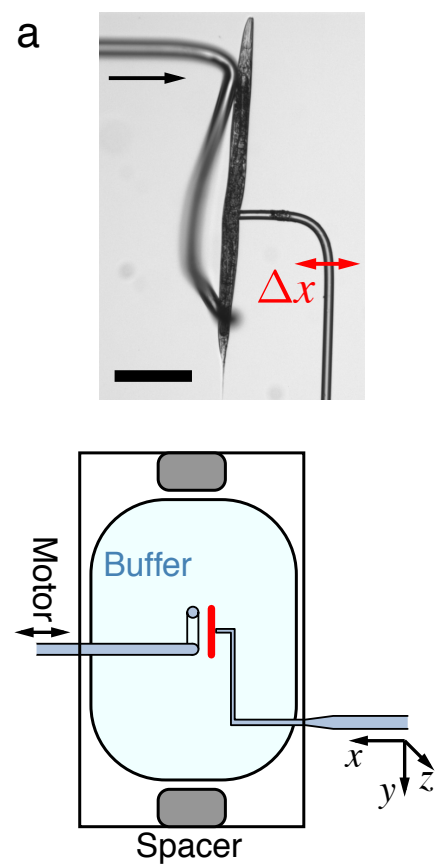

b
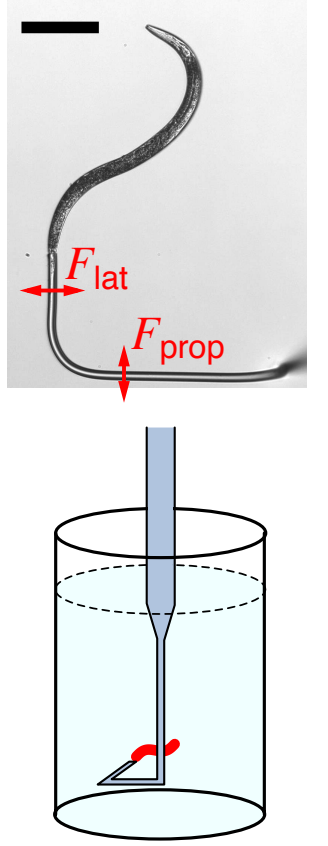

C
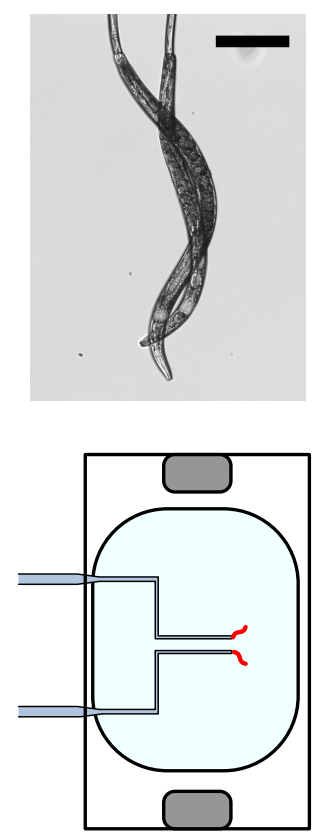

Figure 1 | Principles of the MFS technique as exemplified with the nematode $C$. elegans. Optical images are shown at the top (scale bars, $200 \mu \mathrm{m}$ ), and schematic drawing of the setup (not to scale) at the bottom of each panel. (a) The material properties can be studied through three-point bending measurements on anesthetized worms. The support is mounted to a motor on the left and the force-calibrated micropipette is mounted onto a $x y z$-translational stage on the right. Using suction, the nematode is caught by its side. The support is pushed onto the worm, causing a pipette deflection $\Delta x$ to the right and an antiparallel force $F=$ $k \Delta x$ to act on the body, where $k$ is the spring constant of the pipette. By measuring the bending of the body as a function of applied force, the viscoelastic material properties can be determined [4, 59]. (b) Simultaneous measurements of the lateral $\left(F_{\text {lat }}\right)$ and propulsive $\left(F_{\text {prop }}\right)$ drag forces acting on swimming nematodes held by their tails $[7,13,14]$. (c) The interaction forces between swimming microorganisms can be directly measured with MFS by bringing the tail-tethered swimmers close together. In this experiment, the nematodes formed different, active tangles instead of swimming collectively [60]. 


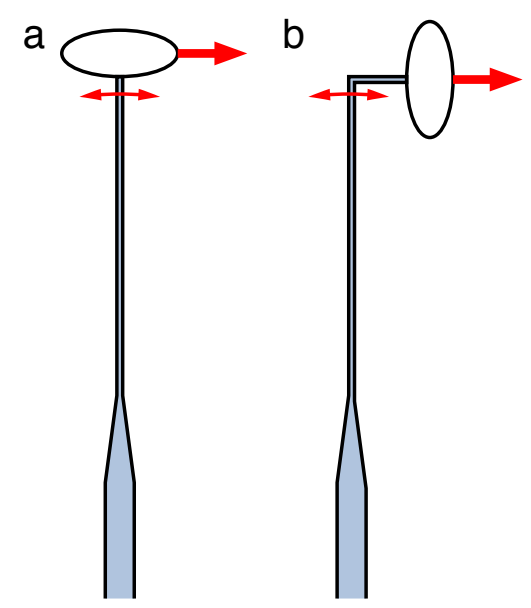

Figure 2 | Basics of the micropipette design. The pipette deflection and the applied force are illustrated with a thin and thick arrow, respectively. (a) If the force is applied perpendicular to the pipette end, the pipette can be completely straight. (b) If the force is applied parallel to the pipette end, a straight corner bend is needed to measure the force from the deflection of the cantilever.

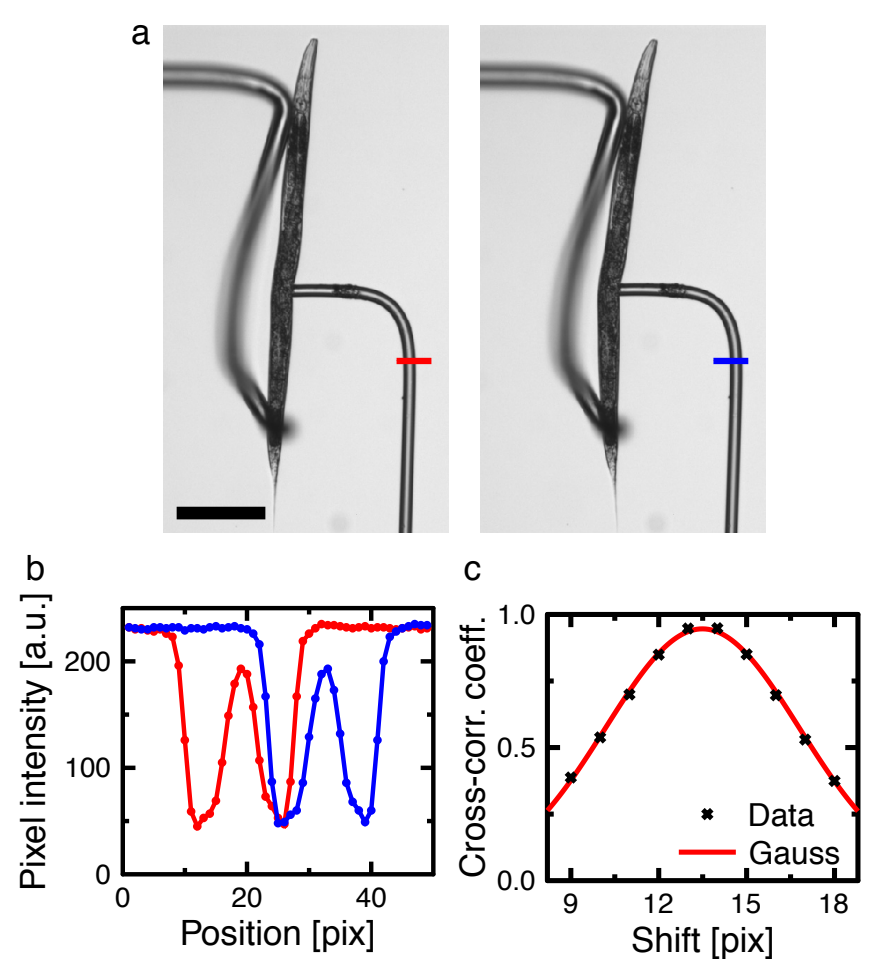

Figure 3 | The cross-correlation image analysis of the micropipette deflection. (a) A line is drawn perpendicularly across the cantilever in the optical images for all frames (two example frames shown). Scale bar $200 \mu \mathrm{m}$. (b) The intensity profile as a function of position along the fixed line plotted for the two examples in (a). Pixel size, $0.95 \mu \mathrm{m}$. (c) A cross-correlation function is used to calculate the shift between the two profiles. The sub-pixel pipette deflection is determined from the mean of a Gaussian fit to the crosscorrelation coefficient as a function of shift. 


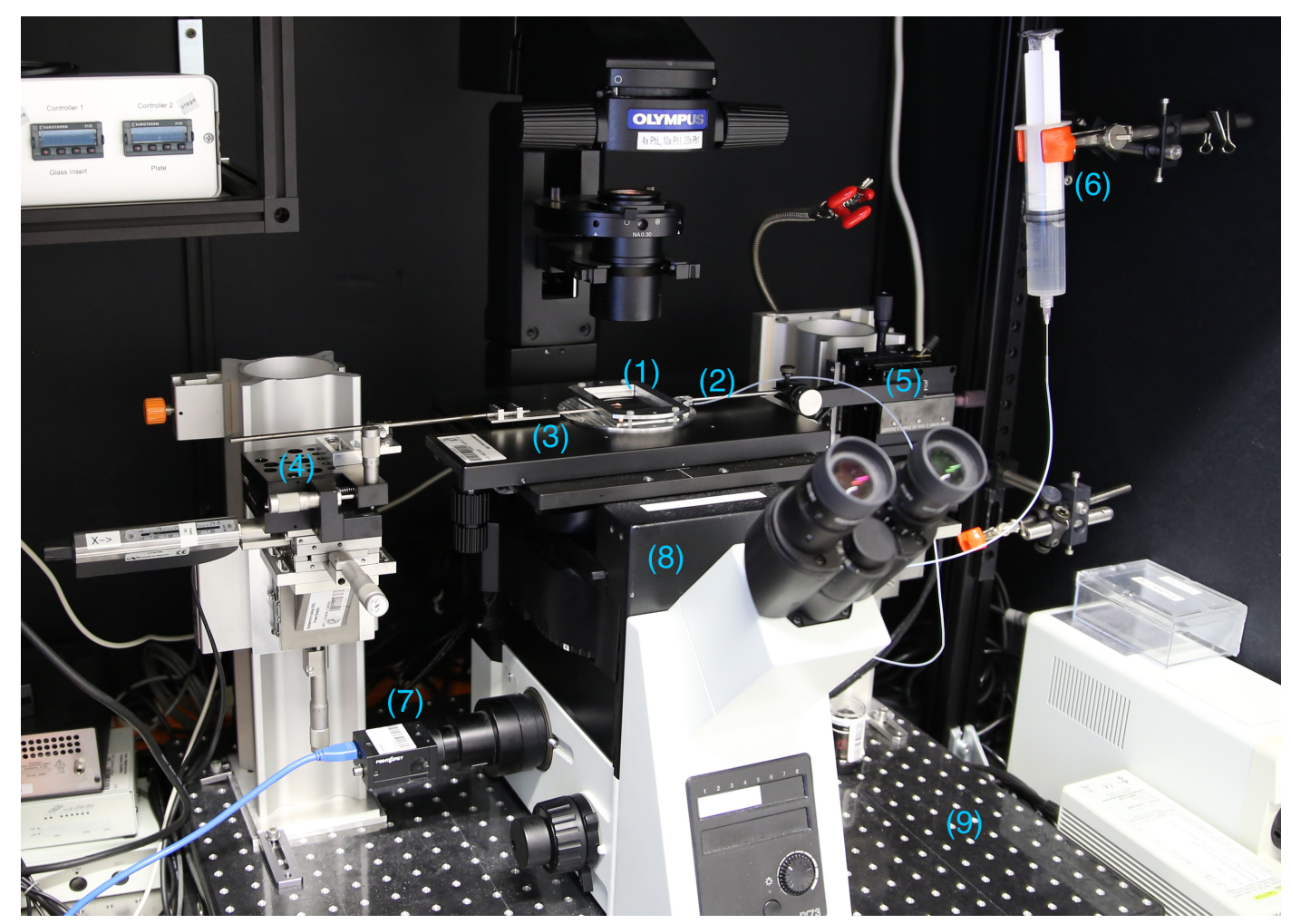

Figure 4 | Equipment setup. The MFS setup is based on an inverted optical microscope (8), which is placed on an active antivibration stage (9). The sample is contained in a liquid cell (1) and held with a micropipette, which is attached to a micropipette holder (2). A substrate holder (3) enters from the opposite side. For controlling the substrate position, the substrate holder is attached to linear stages (4) and/or piezo drives. The micromanipulator set (5) allows for controlling the micropipette position. A syringe (6) is connected to the micropipette to create suction pressure. A scientific camera (7) is used to record image sequences. 


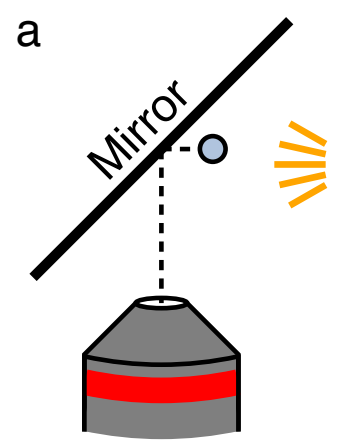

b
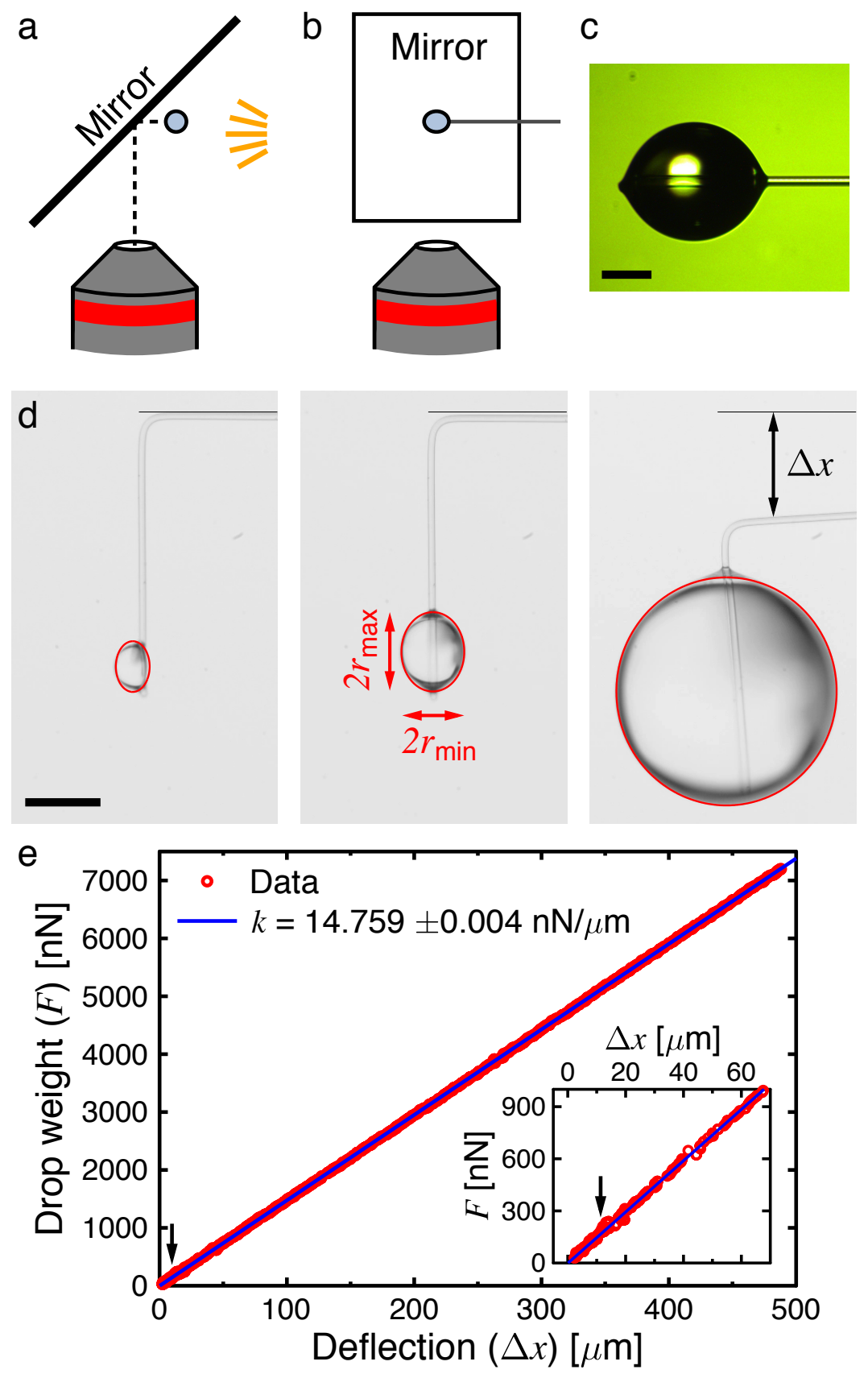

Figure 5 | Micropipette calibration approach as described in Step 9 Option A. (a-b) Schematic drawing of the experimental calibration setup using the mirror approach and a straight micropipette as viewed parallel (a) and perpendicular (b) to the cantilever. The mirror is mounted at a 45-degree angle to the objective and the mirror image of the micropipette is viewed, so that the vertical deflection can be observed from below. External light is applied from the side. (c) Example of an optical image captured with the mirror setup when calibrating a straight micropipette with a water droplet. Scale bar, $50 \mu \mathrm{m}$. (d) Time lapse optical images using an alternative setup (not shown) where a camera is mounted sideways next to the micropipette so that this can be viewed from the side. A small water droplet is used as the weight to deflect the micropipette $(\Delta x)$ from its force-free position (solid black line). The weight is determined as $F=\rho V \mathrm{~g}$, where $\rho$ is the density of the liquid and $V=4 / 3 \pi r_{\min } r_{\min } r_{\max }$ is the volume of the drop when modeled as an ellipsoid (red outline 
around the drop from the edge detection code) with a minimum and maximum radius of $r_{\min }$ and $r_{\max }$. Scale bar, $100 \mu \mathrm{m}$. (e) The drop weight plotted as a function of pipette deflection. The slope of the linear fit corresponds to the pipette spring constant, $k$, and the error corresponds to the $95 \%$ confidence interval of the fit. The experiment was repeated three times and the average spring constant was determined to be $k=$ $14.77 \pm 0.01 \mathrm{nN} / \mu \mathrm{m}$, where the error is the standard deviation. The inset shows a zoom-in on the data close to the origin. The pictures in (d) correspond to the very first (left frame) and last (right frame) data points in the main graph, as well as the one denoted with arrows in both of the graphs (middle frame).
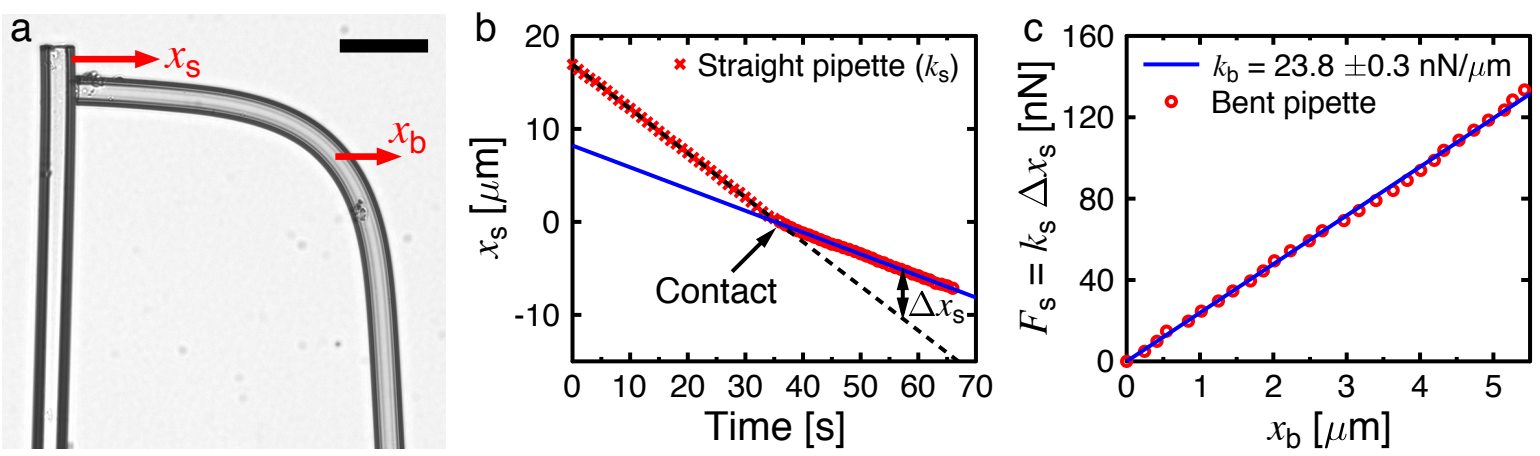

Figure 6 | Micropipette calibration approach as described in Step 9 Option B. (a) Using a straight ("s") pipette, calibrated using the "Calibration A" approach, the spring constant of a bent pipette ("b") can be measured by pushing the two pipettes together with a constant speed and measuring their deflections $\left(x_{\mathrm{s}}\right.$ and $x_{\mathrm{b}}$ ). Scale bar, $20 \mu \mathrm{m}$. (b) The deflection of the straight pipette as a function of time before and after it makes contact with the bent pipette. The relative deflection $\left(\Delta x_{\mathrm{s}}\right)$ is recorded. (c) The force applied by the straight pipette $\left(F_{\mathrm{s}}=k_{\mathrm{s}} \Delta x_{\mathrm{s}}\right)$ on the bent pipette as a function of the bent pipette deflection. The slope of the linear fit gives the spring constant of the bent pipette. The error is the $95 \%$ confidence interval of the fit.
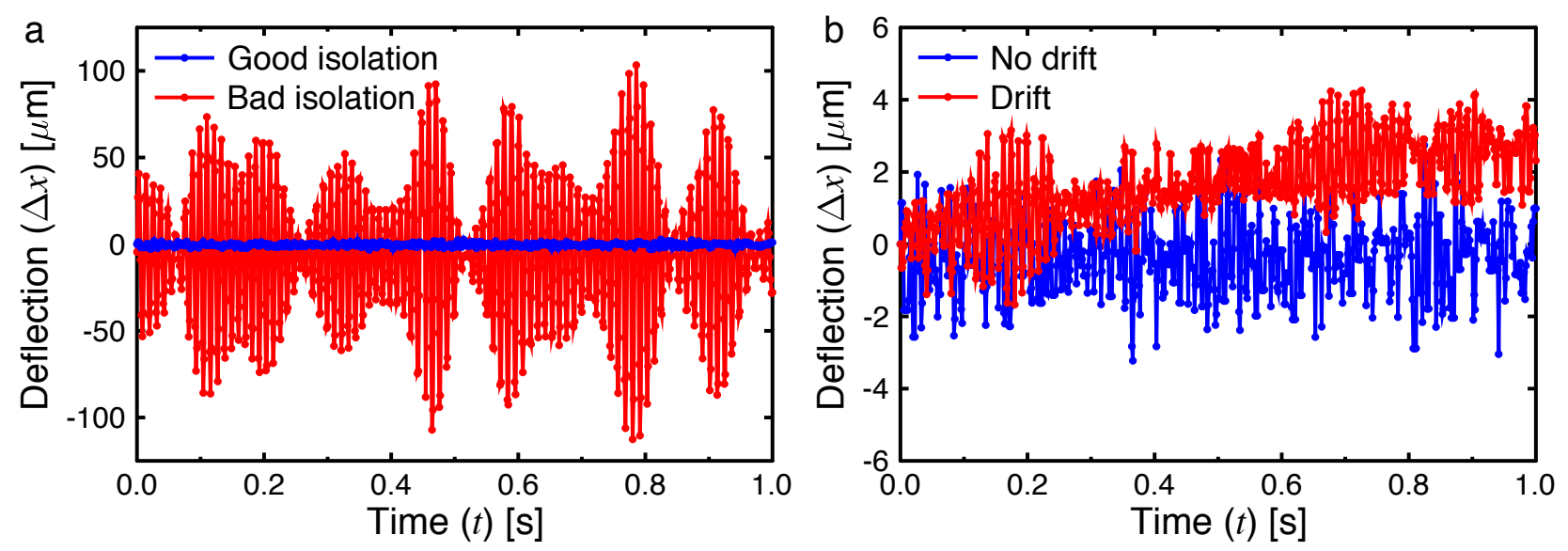

Figure 7 | Examples of typical experimental problems. The micropipette deflection as a function of time for a flexible $(k=3.3 \pm 0.2 \mathrm{nN} / \mu \mathrm{m})$ micropipette in air with problems of (a) bad vibrational isolation and (b) drift. The blue data is the result of a successful experiment on a properly built setup with micropipette fluctuations of less than $\Delta x \sim \pm 2 \mu \mathrm{m}$ in air. Note that in aqueous environments micropipette fluctuations are 
strongly damped, yielding a deflection noise of typically only a few tens of nm with regard to the cantilever's equilibrium position (using an active vibration isolation table).
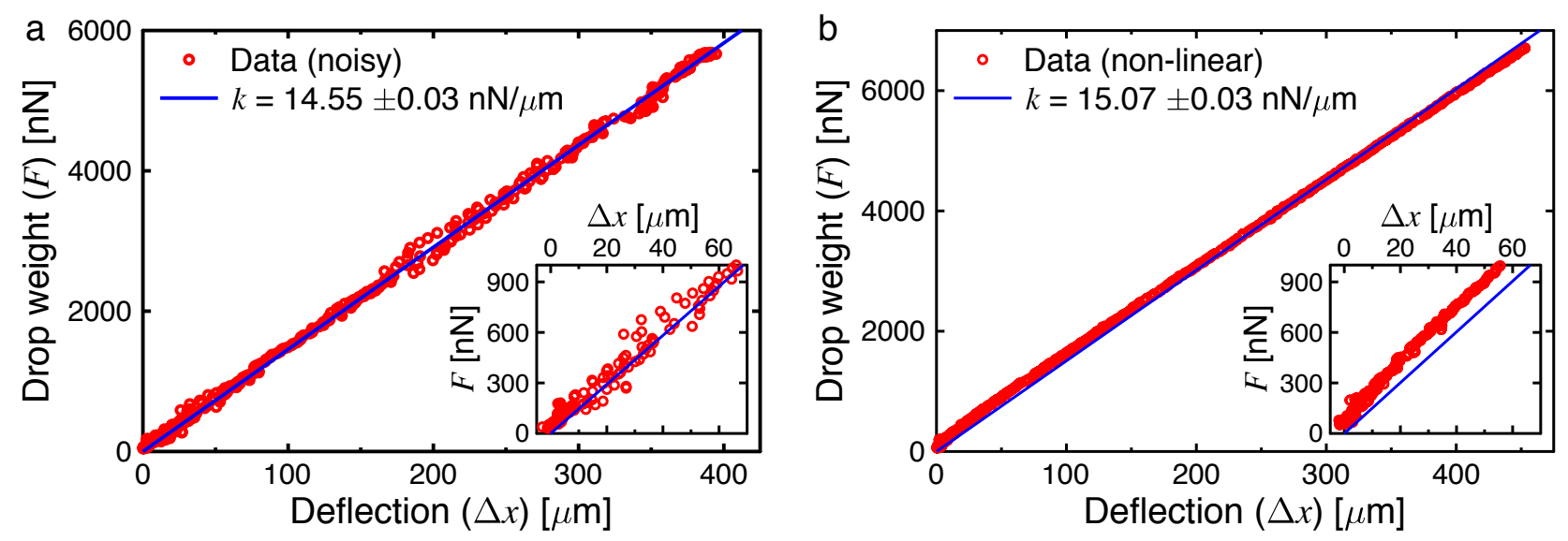

Figure 8 | Examples of two unsuccessful calibration attempts. The drop weight plotted as a function of micropipette deflection from experiments where the data is (a) noisy and (b) non-linear. The solid line shows the linear fit through origin and the error corresponds to the $95 \%$ confidence interval of the fit. In the inset, a zoom-in on the same data at small forces and deflections is shown. See Figure 5e for a comparison with data from a successful calibration of the same pipette (with a correct spring constant of $k=14.77 \pm 0.01 \mathrm{nN} / \mu \mathrm{m}$, where the error is the standard deviation from three successful experiments).
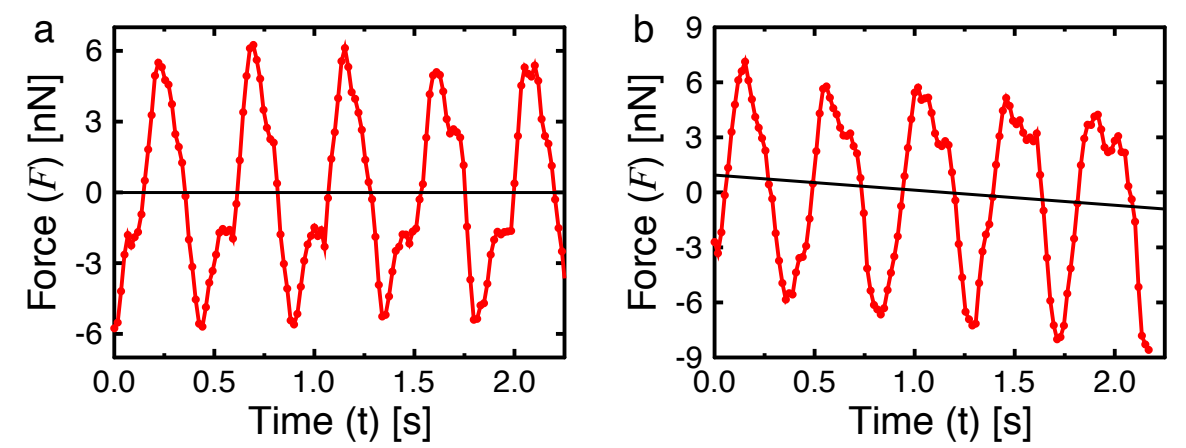

Figure 9 | Anticipated results from C. elegans swimming experiments. (a) Data set (force versus time) from a successful swimming experiment. (b) Results from an experiment that requires troubleshooting due to drift of the pipette, which makes a quantitative force measurement difficult. The black solid lines are linear fits to the data to highlight the drift.

\section{SUPPLEMENTARY INFORMATION}

- Supplementary Video 1: Real-time movie (SuppVid1.mov) from a MFS measurement of a swimming C. elegans nematode (in a 10\% (wt/vol) PEO-M9 buffer solution) using a threedimensional micropipette to measure both the lateral and propulsive drag forces. 
- Supplementary Software 1: Matlab-code "calibration.m" for determining the pipette spring constant in Calibration Option A.

- Supplementary Software 2: Matlab-code "deflection.m" for analyzing the pipette deflection using a cross-correlation approach. 